\title{
Genome-Wide Small RNA Sequencing Identifies MicroRNAs Deregulated in Non-Small Cell Lung Carcinoma Harboring Gain-of-Function Mutant p53
}

\author{
Arindam Datta ${ }^{1,+}{ }^{+}$Pijush Das ${ }^{1,+}$, Sanjib Dey ${ }^{1}$, Sangeeta Ghuwalewala ${ }^{1}$, Dishari Ghatak ${ }^{1}$, \\ Sk. Kayum Alam ${ }^{1}$, Raghunath Chatterjee ${ }^{2, *}$ and Susanta Roychoudhury ${ }^{3, * \text { (D) }}$ \\ 1 Cancer Biology and Inflammatory Disorder Division, CSIR-Indian Institute of Chemical Biology, 4, \\ Raja S.C. Mullick Road, Jadavpur, Kolkata 700032, India; hereisarindam@gmail.com (A.D.); \\ topijush@gmail.com (P.D.); sanby19@gmail.com (S.D.); sangeetaghuwalewala@gmail.com (S.G.); \\ dishari18@gmail.com (D.G.); kayumalam86@gmail.com (S.K.A.) \\ 2 Human Genetics Unit, Indian Statistical Institute, 203 B. T. Road, Kolkata 700108, India \\ 3 Saroj Gupta Cancer Centre and Research Institute, Mahatma Gandhi Road, Thakurpukur, \\ Kolkata 700063, India \\ * Correspondence: raghuchatterjee@gmail.com (R.C.); susantarc@gmail.com (S.R.); \\ Tel.: +91-33-25753243 (R.C.); +91-033-2453-2781/82/83 (S.R.) \\ + These authors contributed equally to this work.
}

Received: 24 August 2019; Accepted: 12 October 2019; Published: 28 October 2019

\begin{abstract}
Mutations in the TP53 gene are one of the most frequent events in cancers. Certain missense mutant p53 proteins gain oncogenic functions (gain-of-functions) and drive tumorigenesis. Apart from the coding genes, a few non-coding microRNAs (miRNAs) are implicated in mediating mutant p53-driven cancer phenotypes. Here, we identified miRNAs in mutant $\mathrm{p} 53^{\mathrm{R} 273 \mathrm{H}}$ bearing non-small cell lung carcinoma (NSCLC) cells while using small RNA deep sequencing. Differentially regulated miRNAs were validated in the TCGA lung adenocarcinoma patients with p53 mutations and, subsequently, we identified specific miRNA signatures that are associated with lymph node metastasis and poor survival of the patients. Pathway analyses with integrated miRNA-mRNA expressions further revealed potential regulatory molecular networks in mutant p53 cancer cells. A possible contribution of putative mutant p53-regulated miRNAs in epithelial-to-mesenchymal transition (EMT) is also predicted. Most importantly, we identified a novel miRNA from the unmapped sequencing reads through a systematic computational approach. The newly identified miRNA promotes proliferation, colony-forming ability, and migration of NSCLC cells. Overall, the present study provides an altered miRNA expression profile that might be useful in biomarker discovery for non-small cell lung cancers with TP53 mutations and discovers a hitherto unknown miRNA with oncogenic potential.
\end{abstract}

Keywords: microRNA; non-small cell lung carcinoma; mutant p53; gain of function; small RNA sequencing; TCGA; novel miRNA; node metastasis; survival

\section{Introduction}

Mutations in the tumor suppressor TP53 gene influence cancer progression and clinical outcomes of human cancers [1,2]. Most of these mutations are monoallelic missense point mutations that result in the synthesis of full length mutant p53 proteins with altered functions [3]. These mutations generally occur at high frequencies in six "hot spot" amino acid residues [3] of the central DNA-binding domain of p53, which causes a loss of its sequence-specific DNA-binding activity. In addition to the loss of wild-type tumor suppressor properties, mutant p53 gains new functions (GOFs) to promote 
various oncogenic phenotypes, including cancer cell proliferation, increased DNA replication, genomic instability, invasion, metastasis, and increased chemo-resistance [4-8]. GOF mutant p53, designated as an oncogenic transcription factor, can modulate the expression of several genes that are involved in oncogenic processes [9]. By cooperating with other transcription factors, such as NF-Y and Sp1, mutant p53 is recruited to target promoters and it facilitates the transcription of the respective genes [9]. Physical interactions of mutant p53 with tumor suppressors p63 and p73 sequesters these proteins and inhibits the transactivation of their respective target genes $[5,10,11]$. Moreover, in response to DNA damage, GOF mutant p53 transactivates cellular genes by recruiting histone modifiers [12].

Lung cancer is one of the leading causes of cancer-related deaths across the world [13]. Approximately $80 \%$ of all primary lung cancer cases are classified as non-small cell lung cancer (NSCLC) $[14,15]$ and more than 50\% of NSCLC patients generally carry TP53 mutations that predict poor prognosis [14-16]. These findings suggest that TP53 mutation determines malignant progression in NSCLC. Among the six "hot spot" missense point mutations, R273 is one of the most frequently mutated $(6.7 \%)$ residues in human cancers [12], particularly in the NSCLC ( 5\%) (IARC database, http://www-p53.iarc.fr). Mutant p53 ${ }^{\mathrm{R} 273 \mathrm{H}}$ has been reported to confer enhanced chemo-resistance and increased cell migration in NSCLC cell line H1299 [17,18]. Moreover, several in vitro and in vivo studies demonstrated the ability of this p53 mutant to induce GOF properties, such as cancer cell invasion, survival, and proliferation; increased migration; drug resistance; anchorage-independent growth; and, genomic instability [19].

The pivotal role of miRNAs in human cancer is well established. Several oncogenic and tumor-suppressive miRNAs have already been identified [20]. The role of miRNAs in mediating tumor suppressor functions of wild-type p53 is also well documented [21]. Genome-wide studies have identified wild-type p53-regulated miRNAs that contribute to tumor suppression and stress responses [22,23]. Although the link between wild type p53 and miRNA is well established, the role of mutant p53 in regulating cellular miRNAs is still emerging. Donzelli et al. first reported that miR-128b is transcriptionally regulated by mutant $\mathrm{p} 53$ and it confers chemo-resistance to lung cancer cells [24]. Another report demonstrated that, upon DNA damage, down-regulation of miR-223 by GOF p53 ${ }^{\mathrm{R} 175 \mathrm{H}}$ via ZEB-1 (a transcriptional repressor) contributes to chemo-resistance of cultured tumor cells [25]. A few other cellular miRNAs (e.g., let-7i, miR-130b, -27a, and -155) is also implicated in mutant p53-driven cancer cell invasion, metastasis, epithelial-to-mesenchymal transition (EMT), and proliferation [26-29]. These evidences suggest that miRNA is a critical mediator of mutant p53 GOF properties in cancer cells. Therefore, identification of mutant p53-regulated miRNAs on a genome-wide scale is of paramount importance in mutant p53 gain-of-function research.

In this study, using small RNA deep sequencing, we identified GOF mutant $\mathrm{p} 53^{\mathrm{R} 273 \mathrm{H}}$ regulated

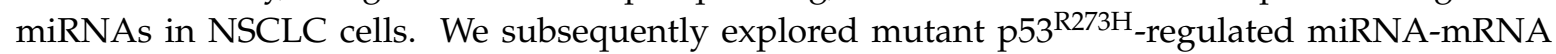
molecular networks and their functions through systematic computational analyses. miRNAs that were obtained from our sequencing experiment were further validated in The Cancer Genome Atlas (TCGA) lung adenocarcinoma patient dataset. Moreover, our analyses identified mutant p53-regulated miRNAs that are associated with lymph node metastasis and poor clinical outcome in lung cancer patients. Most importantly, we discovered a novel miRNA that appeared to be involved in augmenting oncogenic phenotypes in cancer cells. Collectively, the findings of the present study further enrich the evolving knowledge of GOF mutant p53-regulated miRNAs and present a potential novel miRNA with oncogenic functions.

\section{Materials and Methods}

\subsection{Cell Culture}

To generate mutant $\mathrm{p} 53^{\mathrm{R} 273 \mathrm{H}}$ expressing stable $\mathrm{H} 1299$ cell line, the cells were first transfected with pCMV-p53R273H expression plasmid (pCMV-Neo-Bam-p53 R273H, kindly provided by Bert Vogelstein, Johns Hopkins Kimmel Cancer Center, Baltimore, MD, USA) while using Lipofectamine 2000 
(Invitrogen, Thermo Fisher Scientific Inc., Waltham, MA, USA). Forty-eight hours post-transfection, the cells were sub-cultured at a ratio of 1:6 density in media supplemented with G418 (Life Technologies, Thermo Fisher Scientific Inc., Waltham, MA, USA) at a final concentration of $800 \mu \mathrm{g} / \mathrm{mL}$. After 15-20 days, G418 resistant colonies were selected and propagated in G418 containing $(400 \mu \mathrm{g} / \mathrm{mL})$ medium to generate mutant $\mathrm{p} 53^{\mathrm{R} 273 \mathrm{H}}$ expressing stable cell lines. H1299 cells that were infected with an empty vector were kindly provided by Varda Rotter (Weizmann Institute of Science, Rehovot, Israel). A549 cell line was purchased from ATCC. All cell lines were cultured in RPMI 1640 medium (Life Technologies, Thermo Fisher Scientific Inc., Waltham, MA, USA) supplemented with 10\% fetal calf serum, 1\% Pen-Strep, and 0.006\% Gentamicin (Life Technologies, Thermo Fisher Scientific Inc., Waltham, MA, USA). STR profiling confirmed the isogeneity of H1299/EV and H1299/ mutant p53 ${ }^{\mathrm{R} 273 \mathrm{H}}$ cell lines, as described previously [6].

\subsection{Western Blotting}

Cells that were grown in $35 \mathrm{~mm}$ dish were lysed in NP-40 cell lysis buffer (Life Technologies, Thermo Fisher Scientific Inc., Waltham, MA, USA) supplemented with protease inhibitor cocktail (Sigma Aldrich, St. Louis, USA) and $30 \mu \mathrm{g}$ of total protein was immunoblotted with antibodies against p53 (DO-1 (SC-126), Santa Cruz Biotechnology, CA, USA), and $\beta$-actin (\# A5316, Sigma Aldrich, St. Louis, MO, USA).

\subsection{RNA Isolation, Small RNA Library Preparation, and Deep Sequencing}

Small RNA fractions from cells were isolated while using PureLink miRNA Isolation kit (Ambion, Austin, TX, USA) according to the manufacturer's protocol. Briefly, in a two-column based purification method, the cells were first resuspended in binding buffer containing guanidine isothiocyanate and then mixed with ethanol (final concentration 35\%). It was then passed through a spin column and the flow through containing the small RNAs was collected. Ethanol (final concentration 70\%) was added to the flow, followed by extraction though silica-based columns, where the small RNAs bind to the silica membrane. The impurities were subsequently removed by washing the columns with wash buffer and small RNAs were eluted in RNase free water. The enrichment of miRNAs in small RNA samples was assessed by Qubit @2.0 Fluorometer (Life Technologies, Thermo Fisher Scientific Inc., Waltham, MA, USA) and Agilent®2100 Bioanalyzer using Small RNA kit (Agilent Technologies, Santa Clara, CA, USA) (Supplemental Figure S1A). Total RNA-Seq kit V2.0 (Life Technologies, Thermo Fisher Scientific Inc., Waltham, MA, USA) was used to prepare small RNA cDNA libraries. Briefly, small RNAs ( 150-250 ng) were first mixed with Hybridization Solution and Ion Adaptor Mix v2, followed by hybridization in a thermal cycler. Ligation enzyme mix was added to the hybridization reactions and then incubated in a thermal cycler at $16^{\circ} \mathrm{C}$ for $16 \mathrm{~h}$. Adapter-ligated small RNA samples were subsequently reverse transcribed with Ion RT Primer v2 and SuperScript@III Enzyme Mix to make cDNAs. The cDNA products were purified and size-selected while using the magnetic bead clean-up module and PCR amplified with Ion $5^{\prime}$ and $3^{\prime}$ PCR Primers. The amplified DNAs were quantified and assessed for size distribution using Agilent®DNA 1000 Kit in Agilent $(2100$ Bioanalyzer. The cDNA libraries were clonally amplified on Ion Sphere ${ }^{\mathrm{TM}}$ Particles (ISPs) by emulsion PCR in Ion OneTouch ${ }^{\mathrm{TM}}$ System using Ion OneTouch ${ }^{\mathrm{TM}} 200$ Template Kit v2 DL (Life Technologies, Thermo Fisher Scientific Inc., Waltham, MA, USA). The enrichment of the template positive ion spheres was carried out in Ion OneTouch ${ }^{\mathrm{TM}}$ Enrichment System. Template enriched ion sphere particles were subsequently sequenced in Ion PGM ${ }^{\mathrm{TM}}$ sequencer (Model No. 508-U001, Life Technologies, Thermo Fisher Scientific Inc., Waltham, MA, USA) using Ion $316^{\mathrm{TM}}$ Chip and Ion PGM ${ }^{\mathrm{TM}} 200$ Sequencing Kit (Life Technologies, Thermo Fisher Scientific Inc., Waltham, MA, USA). The Torrent Suite Software (Life Technologies, Thermo Fisher Scientific Inc., Waltham, MA, USA) analysis pipeline was used to process raw data that were acquired from the Ion $\mathrm{PGM}^{\mathrm{TM}}$ sequencer to generate read files containing high-quality bases and output base calls in FASTQ file formats. 
Raw sequence reads, as FASTQ format, were pre-processed using FASTX-toolkit (http://hannonlab. cshl.edu/fastx_toolkit/) to generate high-quality reads. Reads were trimmed at $3^{\prime}$ end and the base quality value was calculated while using the Phred quality score $(Q \geq 20)$. We only considered sequencing reads of length between $17 \mathrm{bp}$ to $35 \mathrm{bp}$ (Supplemental Figure S1B). High-quality raw reads were aligned to the reference miRBase (miRBase20; http://www.mirbase.org/) while using SHRiMP aligner (http://compbio.cs.toronto.edu/shrimp/). After alignment, an in-house Perl script was used for counting the reads that were aligned to miRNA, tRNA, rRNA, and adapter sequences. Reads not aligned in SHRiMP aligner were used for further mapping to the human genome (GRCh37/hg19; https://genome.ucsc.edu/) while using Novoalign with miRNA mode (http://www.novocraft.com/). The miRNA read counts were adjusted according to the aligned reads from both SHRiMP and Novoalign. miRNAs with three or more reads were used for differential expression analysis in $\mathrm{R}$ package DESeq. The remaining reads that were mapped to the genome beyond the annotated miRNAs were further used for novel miRNA prediction.

\subsection{Quantitative Real Time PCR}

Total RNA from cell lines was isolated using TRIZOL (Invitrogen, Thermo Fisher Scientific Inc., Waltham, MA, USA), according to the manufacturer's protocol. Around $250 \mathrm{ng}$ of total RNA was reverse transcribed to prepare cDNAs using either miRNA specific stem-loop primers [30] or miScript II RT Kit (Qiagen, Hilden, Germany). Real time PCR reactions of cDNAs were carried out with forward primers specific to each miRNA in 7500 Fast and StepOnePlus Real-Time PCR Systems (Applied Biosystems, Foster City, CA, USA) while using Fast start universal SYBR Green master mix (Roche, Penzberg, Germany). U6 snRNA was used as endogenous reference control. Fold change values $\left(2^{-\Delta \Delta C}\right.$ T $)$ were calculated from the mean of three independent experiments. Two-tailed student's $t$ test was used to compute statistical significance. Primer sequences for mature miRNAs are listed in Supplemental Table S7.

\subsection{Prediction of Novel MiRNAs}

Quality processed reads that were not mapped to miRBase 20 using SHRiMP aligner were further used for novel miRNA prediction. Reads were aligned to the human genome assembly (GRCh37/hg19) while using a Novoalign aligner in miRNA mode to predict the potential miRNA hairpin loci in the genome. The identified loci were subsequently mapped to the miRNA precursor sequences present in miRBase 20. Reads that mapped to known miRNA loci were used to adjust the read count in SHRiMP predicted miRNAs. The remaining reads were used for subsequent analyses in novel miRNA prediction. Candidate sequences that had minimum four read counts across the samples and aligned to loci not reported in miRBase 20 were considered for further analyses. The predicted hairpin sequences were analyzed in mfold 3.2 for secondary structure prediction. Candidate sequences that showed characteristic precursor stem-loop structure with minimum free energy (MFE) $\Delta \mathrm{G} \leq-20 \mathrm{kcal} / \mathrm{mol}$ and formed the stem region of the mature miRNA were considered as potential novel miRNAs.

\section{6. miRNA Target Prediction and Pathway Analysis in IPA}

Differentially regulated miRNAs by mutant $\mathrm{p} 53^{\mathrm{R} 273 \mathrm{H}}$ were analyzed while using the Ingenuity Pathway Analysis (IPA; https://analysis.ingenuity.com) tool to identify their target genes, pathways, and molecular networks. The selected miRNAs were uploaded in IPA and miRNA target filter was used to identify their predicted and experimentally validated targets. Significantly enriched cellular pathways, biological functions, and genetic networks by a given set of miRNAs were generated in IPA. Integrated miRNA-mRNA expression analyses of the differentially regulated miRNAs and target mRNAs in H1299/EV and H1299/mutant p53 ${ }^{\mathrm{R} 273 \mathrm{H}}$ cells were carried out while using the expression pairing tool in IPA. Target genes of novel miRNA were predicted using miRanda [31]. The "seed" sequence of the novel miRNA ( $2-8$ bases at the $5^{\prime}$ end) was aligned to the $3^{\prime}$ UTRs of transcripts 
downloaded from the UCSC GRCh37/hg19 for 100\% complementarily with miRanda score $\geq 150$ and MFE $\leq-20 \mathrm{kcal} / \mathrm{mol}$. The predicted targets were further analyzed in IPA for pathway enrichment.

\subsection{Analysis of Publicly Available MiRNA Datasets}

The TCGA miRNA sequencing dataset of lung adenocarcinoma patients was downloaded from the TCGA data portal. The dataset contained miRNA seq data (level 3) of 230 lung adenocarcinoma patients that were sequenced on Illumina GA and Illumina Hiseq platforms (https://tcga-data.nci. nih.gov/docs/publications/luad_2014/) [32]. The clinical and genetic information of 230 patients were obtained from the article describing the study and cBioPortal (http://cbioportal.org) [32-34]. Lung adenocarcinoma patients were classified based on their TP53 mutation status for validation of the mutant $\mathrm{p} 53^{\mathrm{R} 273 \mathrm{H}}$-regulated miRNAs in TCGA dataset. The types of TP53 mutation present in 230 patients were identified using TP53 somatic mutation dataset in the IARC TP53 Database [2] (version R17, November 2013). Patients harboring truncated p53 were not considered in our analysis because only p53 missense mutants are known to confer GOF properties [35]. Out of 47 differentially regulated miRNAs in H1299/mutant p53 ${ }^{R 273 H}$ cells, we only considered 34 miRNAs that showed read coverage $\geq 2$ in the TCGA dataset. Unsupervised hierarchical clustering of TCGA patient samples was done with complete linkage using Euclidean distance as a distance metrics based on their normalized miRNA read counts to remove the outliers. After outlier elimination, differential expression analysis of miRNAs was carried out between patients with wild-type and missense mutant p53 applying the empirical Bayes, moderated t-statistics in Bioconductor package LIMMA.

Patients were divided into node-positive $\left(\mathrm{N}_{+}, n=85\right)$ and node-negative $\left(\mathrm{N}_{0}, n=138\right)$ groups based on their $\mathrm{N}$ stage status to identify the mutant $\mathrm{p} 53^{\mathrm{R} 273 \mathrm{H}}$ regulated miRNAs that are involved in lymph node metastasis. Patients without $\mathrm{N}$ stage information $(n=7)$ were excluded from the analysis. The Mann-Whitney test was applied to determine the statistical significance of the difference between the relative expression of seven mutant p53-regulated miRNAs in $\mathrm{N}_{+}$and $\mathrm{N}_{0}$ group of patients. Receiver operating characteristic (ROC) analyses were done in GraphPad Prism version 5.03. Survival analysis for seven mutant p53 regulated miRNAs was done using the Kaplan-Meier estimate in GraphPad Prism version 5.03. For survival analysis, we only considered those patients whose survival data was available in the TCGA clinical dataset $(n=195)$ obtained from the cBioPortal and were classified based on high ( $\geq 75$ th percentile) and low ( $\leq 25$ th percentile) expression of the respective miRNA.

NCI-60 miRNA expression dataset (GEO accession number GSE26375) was analyzed to obtain the expression values of the mutant p53-regulated miRNAs. The NCI-60 cell lines were further classified as mesenchymal and epithelial cell types that are based on E-Cadherin/Vimentin expression ratio, as reported by Sun-Mi Park et al [36]. Subsequently, bioconductor package LIMMA was applied to compare the relative expression of these miRNAs between epithelial and mesenchymal groups of NCI-60 cell lines [36].

Small RNA sequencing data of 20 lung adenocarcinoma tissues and 10 squamous cell carcinoma, and 30 corresponding paired noncancerous lung tissues from the same patients were downloaded from the GEO database (GSE33858). Read alignment files (BAM) for these samples were used to identify the expression profile of newly identified miRNA. htseq-count within the HTSeq Python library were used to determine the count of known as well as newly identified miRNAs from both tumor and corresponding noncancerous tissues [37]. Differential expression analysis of miRNAs was performed while using DESeq [38].

\subsection{Cloning of Novel MiRNA}

The newly identified miRNA (miR-X) precursor sequence was amplified from genomic DNA using specific primers having KpnI and HindIII restriction enzyme sites at $5^{\prime}$ and $3^{\prime}$ ends, respectively. Following digestion with KpnI and HindIII, PCR amplified product was cloned into the pRNA-U6.1 
miRNA expression vector. The ligated products were transformed in E.coli $\mathrm{DH} 5 \alpha$ and positive clones were selected by restriction digestion and sequencing.

\subsection{Cell Proliferation Assay}

Cells that were transfected with pRNA-U6.1-miR-X or control empty vector were seeded at 4000 cells/well of 96 well plate and cultured in complete medium up to seven days. Cell proliferation was measured on individual days while using WST1 cell proliferation reagent (Roche, Penzberg, Germany) according to the manufacturer's protocol. Relative viability index was calculated in MS-Excel from the average absorbance values that were obtained from three biological replicates.

\subsection{Intracellular Ki-67 Staining}

Cells that were transfected with pRNA-U6.1 vector or pRNA-U6.1-miR-X plasmid were trypsinized and resuspended in wash buffer (1X PBS containing 1\% FBS and 0.02\% sodium azide) $48 \mathrm{~h}$ post-transfection. Cells were fixed in $1 \%$ paraformaldehyde for $15 \mathrm{~min}$. and then washed with wash buffer followed by permeabilization with 1X Perm 2 solution (BD, Franklin Lakes, New Jersey, USA). The cells were washed with saponin wash buffer and resuspended in saponin wash buffer containing Ki-67 antibody (1:50, D2H10, Rabbit mAB, Cell signaling technology, Danvers, MA, USA) or isotype control (Rabbit $\mathrm{mAb}$ IgG (DA1E) XPR , Cell signaling technology) and incubated for $40 \mathrm{~min}$ at room temperature. The cells were washed and incubated with goat anti-rabbit Alexa Fluor 488 secondary antibody (1:100, Molecular Probes, Invitrogen, Carlsbad, CA, USA) for $30 \mathrm{~min}$ in the dark. The cells were washed three times with saponin wash buffer and finally resuspended in $1 \%$ paraformaldehyde solution. The stained cells were analyzed by FACS in BD LSRFortessa while using BD FACSDiva 6.2 software.

\subsection{Colony Formation Assay}

Around 1500 cells were seeded on $6 \mathrm{~cm}$ dishes and allowed to grow in complete medium for 10-15 days until the appearance of visible colonies. The colonies were stained with $0.1 \%$ methylene blue for $30 \mathrm{~min}$ at room temperature, followed by washing with 1X PBS. Stained colonies were photographed and counted while using ImageJ software (http://rsb.info.nih.gov/ij/). The average number of colonies from three independent biological experiments were plotted in GraphPad Prism version 5.03.

\subsection{Monolayer Wound Healing Assay}

Approximately $1 \times 10^{6}$ cells plated on a six-well plate were transfected with control pRNA-U6.1 vector or pRNA-U6.1-miR-X and they were grown for $30 \mathrm{~h}$ before the scratch was introduced. Photographs were taken at $0 \mathrm{~h}$ and 16-18 $\mathrm{h}$ after the scratch was introduced using a microscope (Olympus $1 \times 51$, camera Jenoptik). The relative distance that was migrated by the cells over time was measured in order to compare the wound healing capability of control and miR-X overexpressing cells. The percentage of average distance migrated by the cells was calculated from the data that were obtained from three independent experiments.

\subsection{Availability of Data and Materials}

Small RNA sequencing data have been submitted to the GEO database with the accession number GSE68353

\section{Results}

\subsection{Small RNA Sequencing Identifies MiRNAs Deregulated in GOF Mutant p53 Cancer Cells}

We carried out small RNA sequencing in NSCLC cell line H1299 either harboring a control empty vector (H1299/EV) or stably expressing mutant p53 ${ }^{\mathrm{R} 273 \mathrm{H}}\left(\mathrm{H} 1299 /\right.$ mutant $\left.\mathrm{p} 53^{\mathrm{R} 273 \mathrm{H}}\right)$ to identify GOF mutant p53-regulated miRNAs on a genome-wide scale (Figure 1A). Normalized read counts of each 
group showed a good correlation between biological replicates with an average Pearson correlation coefficient $>0.9$ (Figure $1 \mathrm{~B}$, Table 1 ). Approximately 72 to $80 \%$ reads were mapped to the reference miRBase (miRBase20; http://www.mirbase.org/) in both H1299/EV and H1299/mutant p53 ${ }^{\text {R273H }}$ cells (Table 1). Differential expression analysis of the mapped miRNAs yielded forty-seven differentially regulated miRNAs between the control and mutant $p 53^{\mathrm{R} 273 \mathrm{H}}$ expressing cells ( $p$-value $<0.05$; fold change $\geq 2$ ) (Figure $1 C$ ). Among these, 21 miRNAs were found to be up-regulated and 26 were down-regulated in H1299/mutant p53 ${ }^{R 273 H}$ cells. Subsequent qRT-PCR-based validation of seven randomly selected miRNAs in the control and mutant $\mathrm{p} 53^{\mathrm{R} 273 \mathrm{H}}$ cells showed concordant results with the genome-wide sequencing data (Figure 1D). The differentially regulated miRNAs were subjected to pathway analysis while using the Ingenuity Pathway Analysis tool (IPA; https://analysis.ingenuity.com) to gain further insight into the biological changes that they could impart in mutant p53 cells. Crucial cellular functions, such as the regulation of cell cycle, cellular development, growth and proliferation, movement, and DNA replication, recombination, and repair were significantly enriched in our analyses (Table 2). Notably, most of the pathways enriched are generally found to be altered in cancer and affected by GOF mutant p53 [4,5]. Three miRNA-mRNA networks (IPA score $\geq 25$ ) were significantly enriched by these miRNAs (Figure 2 and Supplemental Table S1). Among the significantly enriched networks, TP53 was found to either be targeted by, or regulate many, miRNAs that were deregulated in GOF mutant p53 cells (Figure 2). Downregulated miRNAs, like mir-194 and mir-296, were the targets of p53 and possibly works in a positive feedback loop with wildtype p53, while upregulated miRNAs, mir-132, mir-140, and mir-17 were either directly or indirectly regulated by mutant p53. Apart from TP53, ephrin-B family receptor EPHB6, transforming growth factor $\beta$ (TGFBI), and Insulin were also enriched as major focal nodes in the miRNA-mRNA networks (Figure 2). Interactions with EphB6, TGF $\beta 1$, and Insulin suggest the possible contribution of these crucial signaling factors in mutant p53 oncogenic gain-of-functions.

Table 1. Sequencing summary of the small RNA libraries. Raw reads were quality processed and aligned to the reference human genome (GRCh37/hg19) and miRBase (miRBase20). Total number and the percentage of processed reads mapped to miRNA, tRNA, rRNA, and adaptors are shown. The number of miRNAs identified in each sample with $\geq 1$ or $\geq 3$ read counts are indicated.

\begin{tabular}{|c|c|c|c|c|c|}
\hline \multicolumn{2}{|l|}{ Samples } & $\begin{array}{l}\text { H1299/EV } \\
\text { Replicate } 1\end{array}$ & $\begin{array}{l}\text { H1299/EV } \\
\text { Replicate } 2\end{array}$ & $\begin{array}{l}\text { H1299/R273H } \\
\text { Replicate } 1\end{array}$ & $\begin{array}{c}\text { H1299/R273H } \\
\text { Replicate } 2\end{array}$ \\
\hline \multicolumn{2}{|l|}{ Total Raw Reads } & $1,442,378$ & 751,538 & $1,292,998$ & 874,753 \\
\hline \multirow{2}{*}{$\begin{array}{l}\text { Mapping to References (miRBase, } \\
\text { rRNA, tRNA \& Adaptor) }\end{array}$} & Total Reads & 582,481 & 261,368 & 462,283 & 420,018 \\
\hline & Percentage & 77.27 & 76.82 & 81.54 & 83.92 \\
\hline Reads mapped to miRNA & Percentage & 71.65 & 73.78 & 80.06 & 81.53 \\
\hline \multirow{2}{*}{ Reads mapped to tRNA } & Total Reads & 40,071 & 9763 & 7932 & 11,191 \\
\hline & Percentage & 5.32 & 2.87 & 1.40 & 2.24 \\
\hline Reads mapped to rRNA & Total Reads & 2235 & 575 & 406 & 767 \\
\hline Reads mapped to adaptor & Percentage & 0.0040 & 0.0024 & 0.0019 & 0.0010 \\
\hline $\begin{array}{l}\text { Total expressed miRNA ( } 1 \text { or } \\
\text { more reads) }\end{array}$ & & 692 & 512 & 507 & 518 \\
\hline miRNA ( 3 or more reads) & & 363 & 245 & 299 & 306 \\
\hline
\end{tabular}

The expression of miRNAs is anti-correlated with that of their target mRNAs. Therefore, we carried out an integrated miRNA-mRNA expression analysis to identify potential miRNA-mRNA modules through which GOF mutant p53 could impart its oncogenic functions (Figure 3). We determined the anti-correlated miRNA-mRNA modules by integrated analysis of miRNA and mRNA expression in 
H1299 cells expressing mutant $\mathrm{p} 53^{\mathrm{R} 273 \mathrm{H}}$. For this we first analyzed the transcriptome data in H1299 cells with or without R273H mutant p53 [39]. Next, using the miRNA-mRNA expression pairing tool in IPA, we identified anti-correlated miRNA-target mRNA pairs in H1299 cells expressing mutant p53 ${ }^{\mathrm{R} 273 \mathrm{H}}$ (Figure 3). Twelve up-regulated and eight down-regulated miRNAs showed an inverse correlation with the expression patterns of their target genes in presence of mutant $\mathrm{p} 53^{\mathrm{R} 273 \mathrm{H}}$ (Figure 3 and Supplemental Table S2). Genes, like TGFB1, SERPINA1, FOSL1, and SRGAP1, were targeted by more than one miRNA, suggesting their regulation by multiple miRNAs in mutant p53 cancer cells.

A

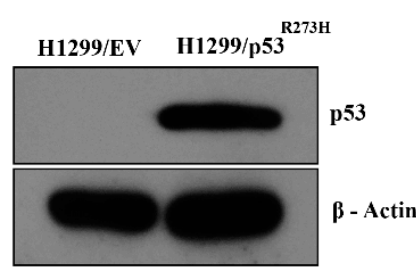

C

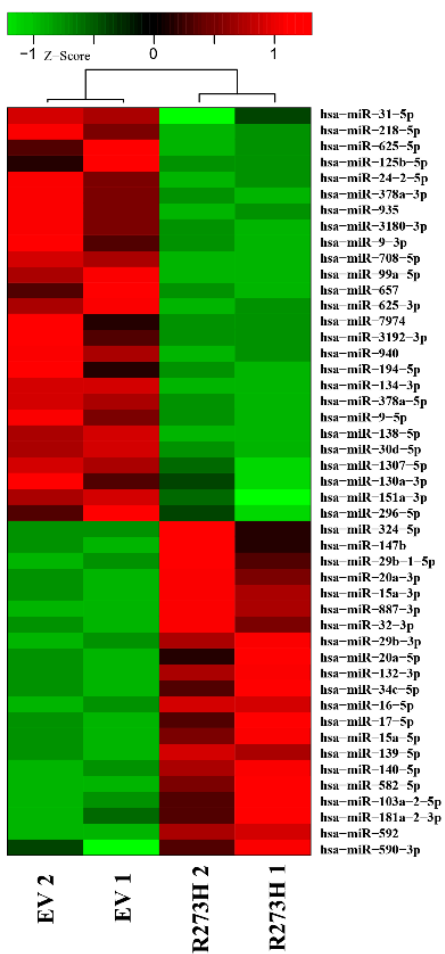

B

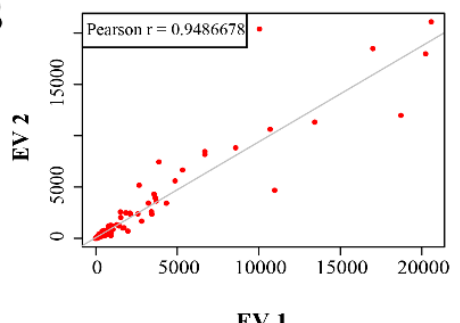

D

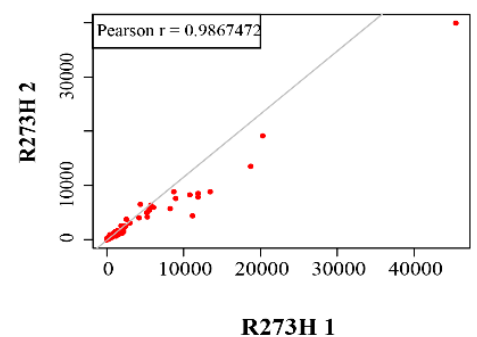

R273H 1

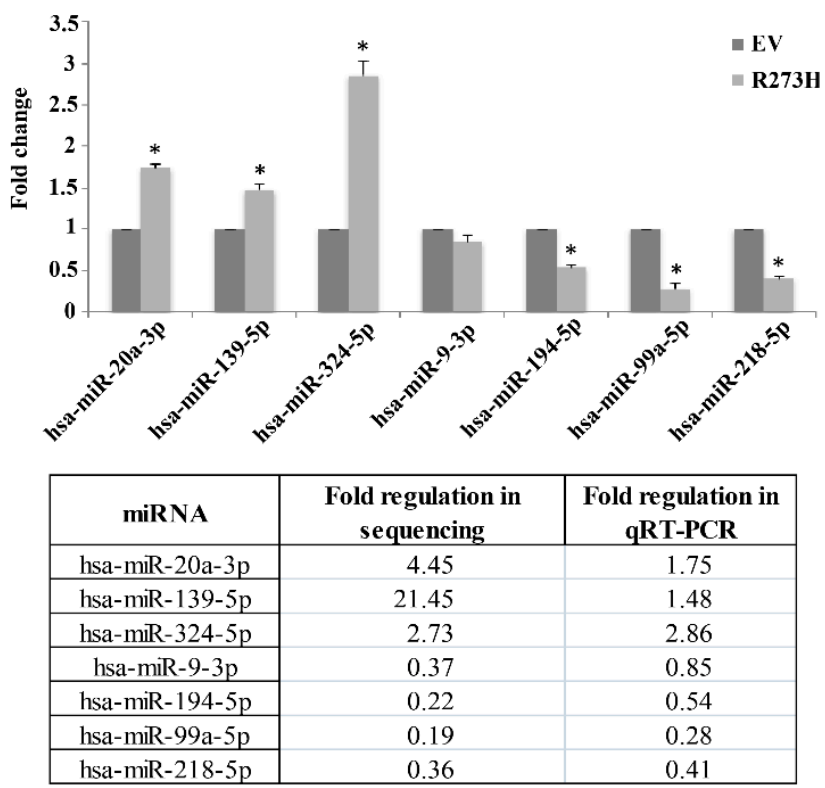

Figure 1. miRNA expression profiling in $\mathrm{H} 1299$ cells expressing mutant $\mathrm{p} 53^{\mathrm{R} 273 \mathrm{H}}$ using small RNA sequencing. (A) Immunoblot showing mutant $\mathrm{p} 53^{\mathrm{R} 273 \mathrm{H}}$ level in $\mathrm{H} 1299 /$ mutant $\mathrm{p} 53^{\mathrm{R} 273 \mathrm{H}}$ stable cells. (B) Scatter plots showing a correlation of normalized read counts between biological replicates of individual samples. (C) Heat map showing normalized read counts of miRNAs differentially expressed ( $p$-value $\leq 0.05$ ) between H1299/mutant $\mathrm{p} 53^{\mathrm{R} 273 \mathrm{H}}$ and H1299/EV cells. Hierarchical clustering of samples is shown. Color bar indicates Z- scores of normalized read counts. Red color indicates high expression, green color indicates low expression. (D) Validation of the selected differentially expressed miRNAs in H1299/mutant p53 ${ }^{\mathrm{R} 273 \mathrm{H}}$ cells using qRT-PCR. Bar graphs represent mean \pm s.d.; $n \geq 2$; two-tailed Student's $t$-test: ${ }^{*} p<0.05$. A relative comparison of qRT-PCR data with the deep sequencing results is shown. 

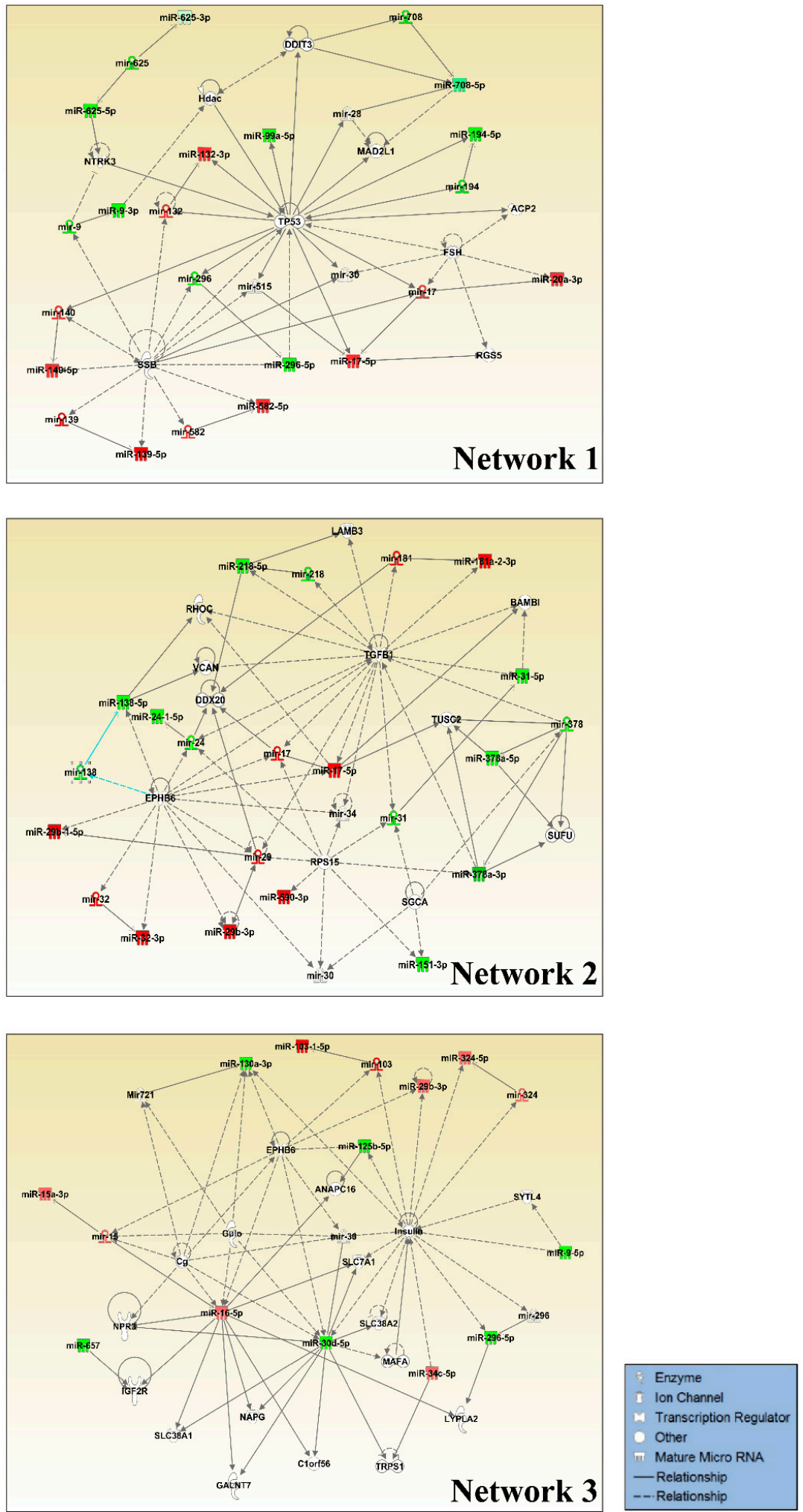

Figure 2. Molecular networks enriched by miRNAs differentially expressed in H1299/mutant p53R273H cells. Ingenuity Pathway Analysis (IPA) generated top three significantly enriched regulatory networks of mutant $\mathrm{p} 53^{\mathrm{R} 273 \mathrm{H}}$ regulated miRNAs. The networks illustrate direct or indirect interactions between altered miRNAs and their target genes. Only the highlighted (green/red) miRNAs were present in our dataset. Green and red represent down-regulated and up-regulated miRNAs, respectively. 
miRNA Up_mRNA Down

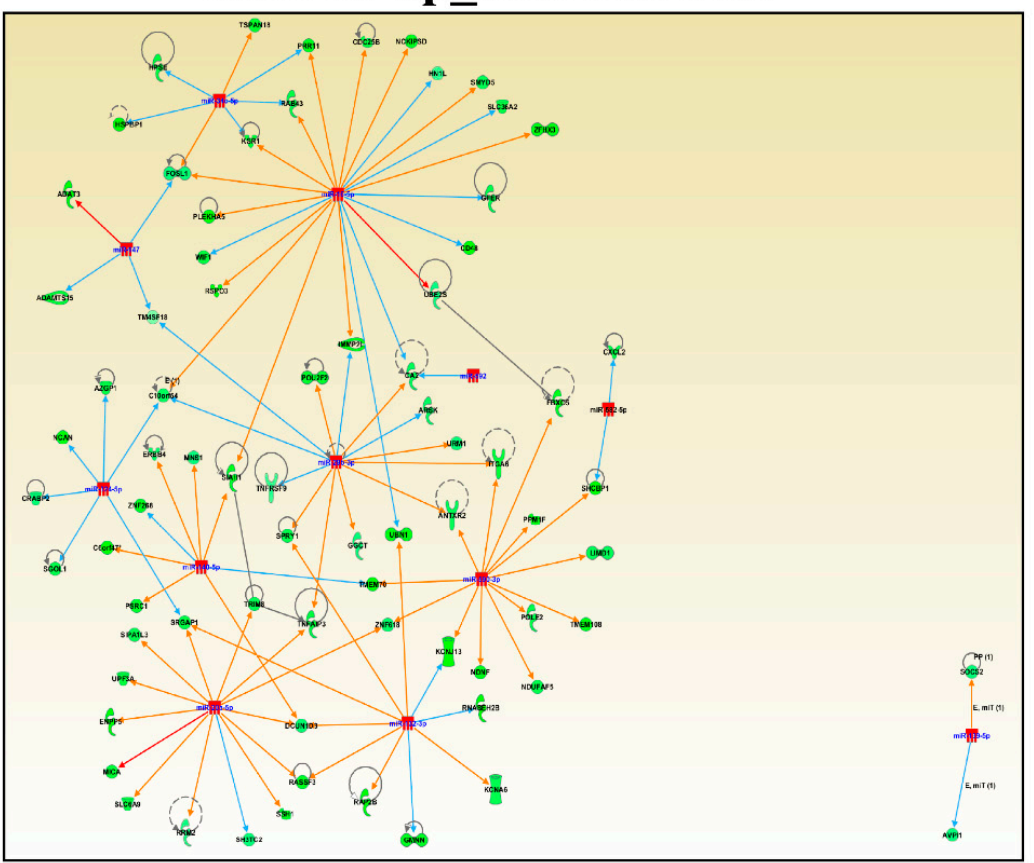

miRNA Down_mRNA Up

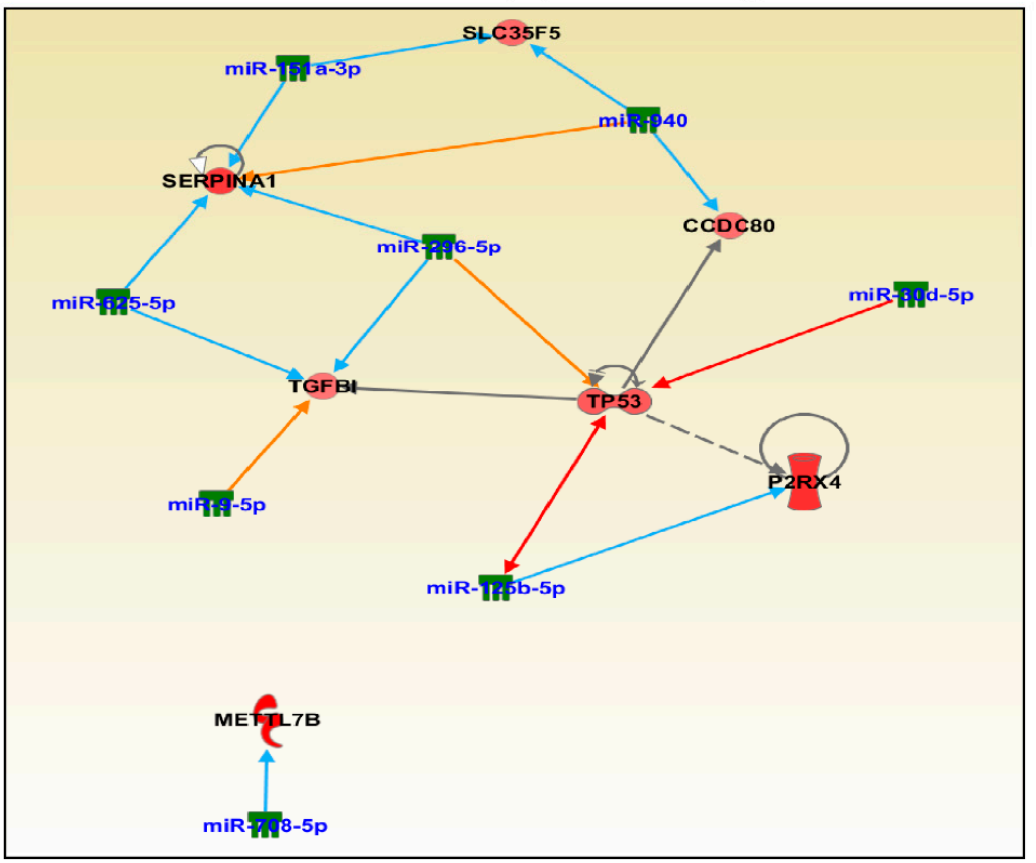

\begin{tabular}{ll|} 
& Enzyme \\
I Ion Channel \\
M. Transcription Regulator \\
Other \\
II. Mature Micro RNA \\
- Relationship \\
--- Relationship
\end{tabular}

Figure 3. Molecular networks of anti-correlated miRNA-mRNA pairs in H1299/mutant p53 ${ }^{\mathrm{R} 273 \mathrm{H}}$ cells derived from the integrated miRNA-mRNA expression analysis. Target genes of the up-regulated miRNAs (Red) were down-regulated (Green) [upper panel] and those of down-regulated miRNAs (Green) were up-regulated (Red) [lower panel] in the presence of mutant $\mathrm{p} 53^{\mathrm{R} 273 \mathrm{H}}$. The color intensity of the nodes indicates relative miRNA or mRNA expression levels in presence of mutant $\mathrm{p} 53^{\mathrm{R} 273 \mathrm{H}}$. The types of interaction between miRNAs and their respective target mRNAs are represented by arrows in dark red (experimentally validated), bright orange (highly predicted), and light blue (moderately predicted). 
Notably, the analyses showed that most of the target genes of mutant $\mathrm{p} 53^{\mathrm{R} 273 \mathrm{H}}$-regulated miRNAs were down-regulated in the mutant p53 cells, which thereby suggests an important role of cellular miRNAs in mediating GOF mutant p53-mediated regulation of cellular gene expression.

Table 2. Molecular and cellular functions enriched in H1299/mutant p53 $^{\mathrm{R} 273 \mathrm{H}}$ cells.

\begin{tabular}{ccc}
\hline \multicolumn{2}{c}{ H1299/EV vs H1299/mutant p53 $^{\text {R273H }}$} & \\
\hline Molecular and Cellular Functions & \\
\hline Name & $p$-Value & \# Molecules \\
\hline Cell Cycle & $7.36 \mathrm{E}-06-4.52 \mathrm{E}-02$ & 8 \\
\hline Cellular Development & $7.50 \mathrm{E}-05-4.82 \mathrm{E}-02$ & 13 \\
\hline Cellular Growth and Proliferation & $7.50 \mathrm{E}-05-4.82 \mathrm{E}-02$ & 12 \\
\hline Cellular Movement & $2.72 \mathrm{E}-04-3.14 \mathrm{E}-02$ & 10 \\
\hline DNA Replication, Recombination and Repair & $3.67 \mathrm{E}-04-4.82 \mathrm{E}-02$ & 5 \\
\hline
\end{tabular}

\subsection{Validation of GOF Mutant p53-Regulated MiRNAs in Lung Adenocarcinoma (LUAD) Patients}

Next, the mutant $\mathrm{p} 53^{\mathrm{R} 273 \mathrm{H}}$-regulated miRNAs that were obtained from our sequencing study were validated in lung adenocarcinoma patients having wild-type or missense mutant p53 from the TCGA LUAD dataset (https://tcga-data.nci.nih.gov/docs/publications/luad_2014/). Seven miRNAs were found to be significantly ( $p$-value $<0.05)$ altered in patients with p53 mutations and showed expression patterns that were similar to that observed in our cell line experiments (Figure 4A). Two miRNAs (miR-132 and -147b) were significantly up-regulated, whereas five (miR-99a, -218, -30d, -24, and -625 ) were found to be significantly down-regulated (Figure 4A,B). The association observed between the expression patterns of these miRNA with p53 mutation status in lung cancer patients might point towards their potential role in mediating mutant p53-driven cancer phenotypes. It is notable, however, that only seven of the mutant $\mathrm{p} 53^{\mathrm{R} 273 \mathrm{H}}$ regulated miRNAs could be successfully validated in TCGA patient dataset. This might be attributed to genetic heterogeneity as well as cell type compositions amongst the patients, a phenomenon that is not generally observed in cell lines. Additionally, the differential effects of various p53 mutants upon miRNAs or target genes cannot be overruled.

The association of p53 mutations with tumor aggressiveness and metastasis, including that in NSCLC, is well demonstrated $[15,40,41]$. Therefore, we sought to determine the possible contribution of these seven mutant $\mathrm{p} 53^{\mathrm{R} 273 \mathrm{H}}$-regulated miRNAs in predicting lymph node metastasis (LNM) in lung adenocarcinoma patients. To this end, the relative expression levels of individual miRNAs were compared between lymph node positive $\left(\mathrm{N}_{+}\right)$and lymph node negative $\left(\mathrm{N}_{0}\right)$ group of patients (Figure 5A and Supplemental Table S3). The up-regulated miRNAs (miR-132 and miR-147b) showed significantly ( $p$-value $\leq 0.05$ ) higher expression in $\mathrm{N}_{+}$patients when compared to the $\mathrm{N}_{0}$ group (Figure 5A), thereby suggesting a possible role of these miRNAs in driving lymph node metastasis in lung cancer. 
A
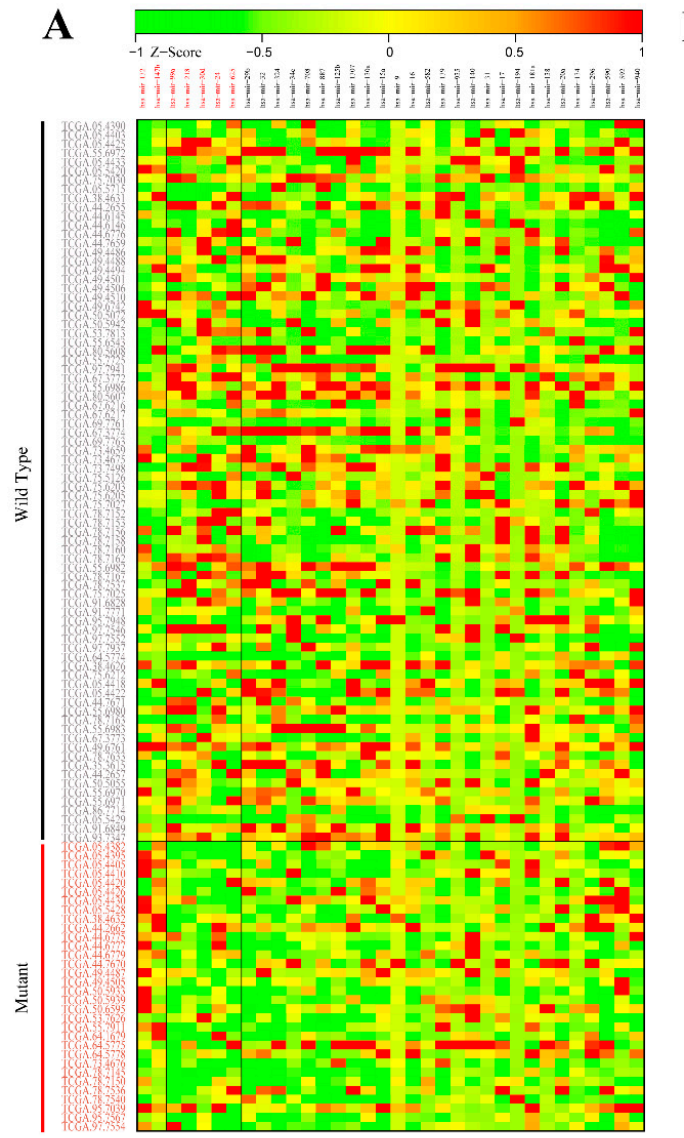

B
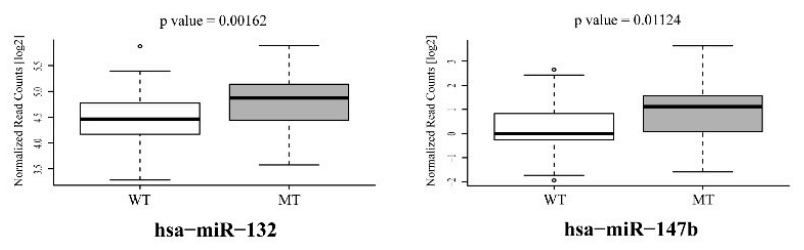

$p$ value $=0.000074$
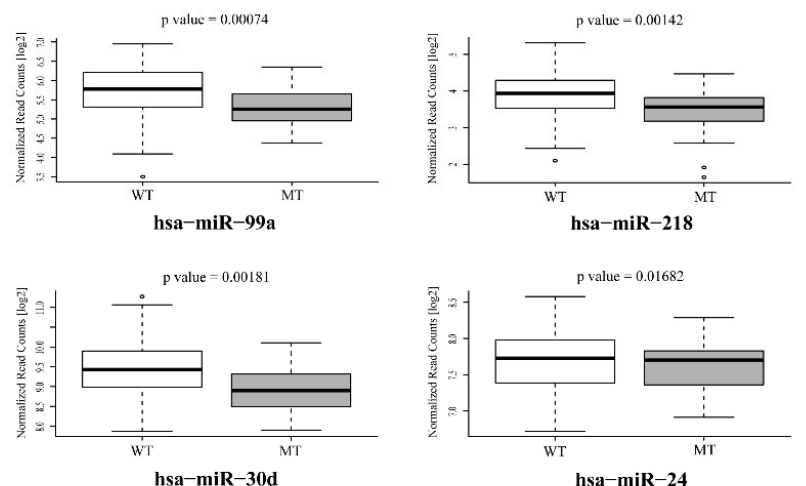

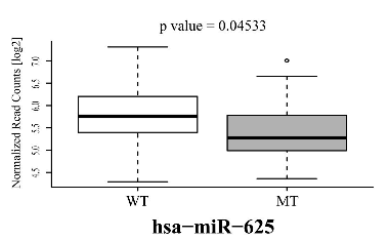

Figure 4. Validation of mutant $\mathrm{p} 53^{\mathrm{R} 273 \mathrm{H}}$-regulated miRNAs in the TCGA lung adenocarcinoma patient dataset. (A) Heat map showing normalized read counts of mutant $\mathrm{p} 53^{\mathrm{R} 273 \mathrm{H}_{\text {-regulated miRNAs across }}}$ TCGA lung adenocarcinoma patients bearing wild type and mutant p53. Based on TP53 mutation status, TCGA patients were categorized into two groups, wild type and mutant p53 patients. The relative expression levels of mutant p53-regulated miRNAs obtained in the present study were subsequently validated by comparing their normalized read counts between these two groups of patients. Patients with wild type p53 and mutant p53 are shown in black and red color letters respectively. miRNAs shown in red are significantly $(p<0.05)$ altered between wild-type and mutant p53 patients with a pattern similar to that of our small RNA sequencing results. Heat map scale bar indicates Z-scores of normalized read count values. (B) Box-Whisker plots showing log2 transformed normalized read counts of two up-regulated and five down-regulated miRNAs in patients with mutant p53. $p$-values are indicated. 
A
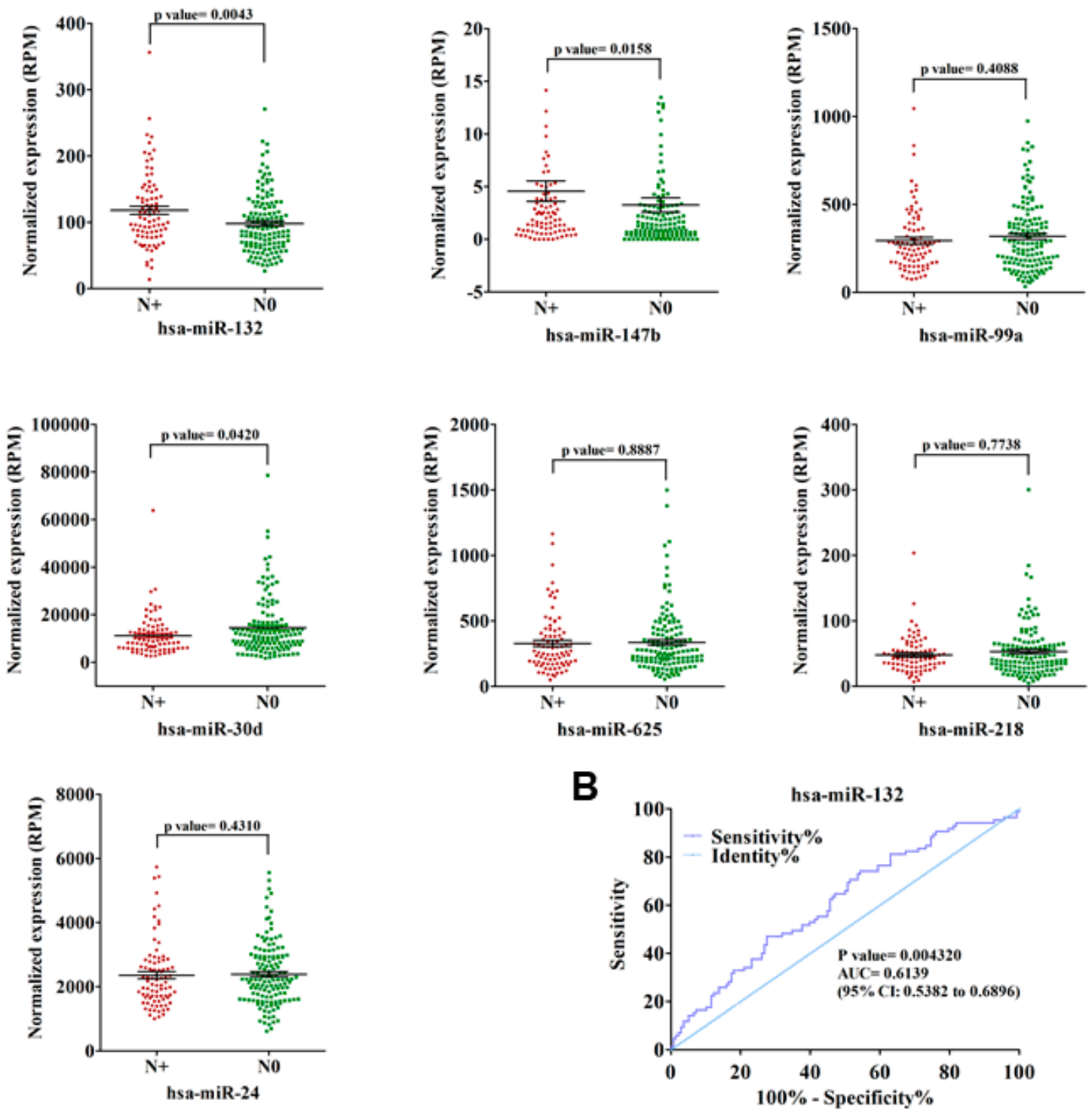

B
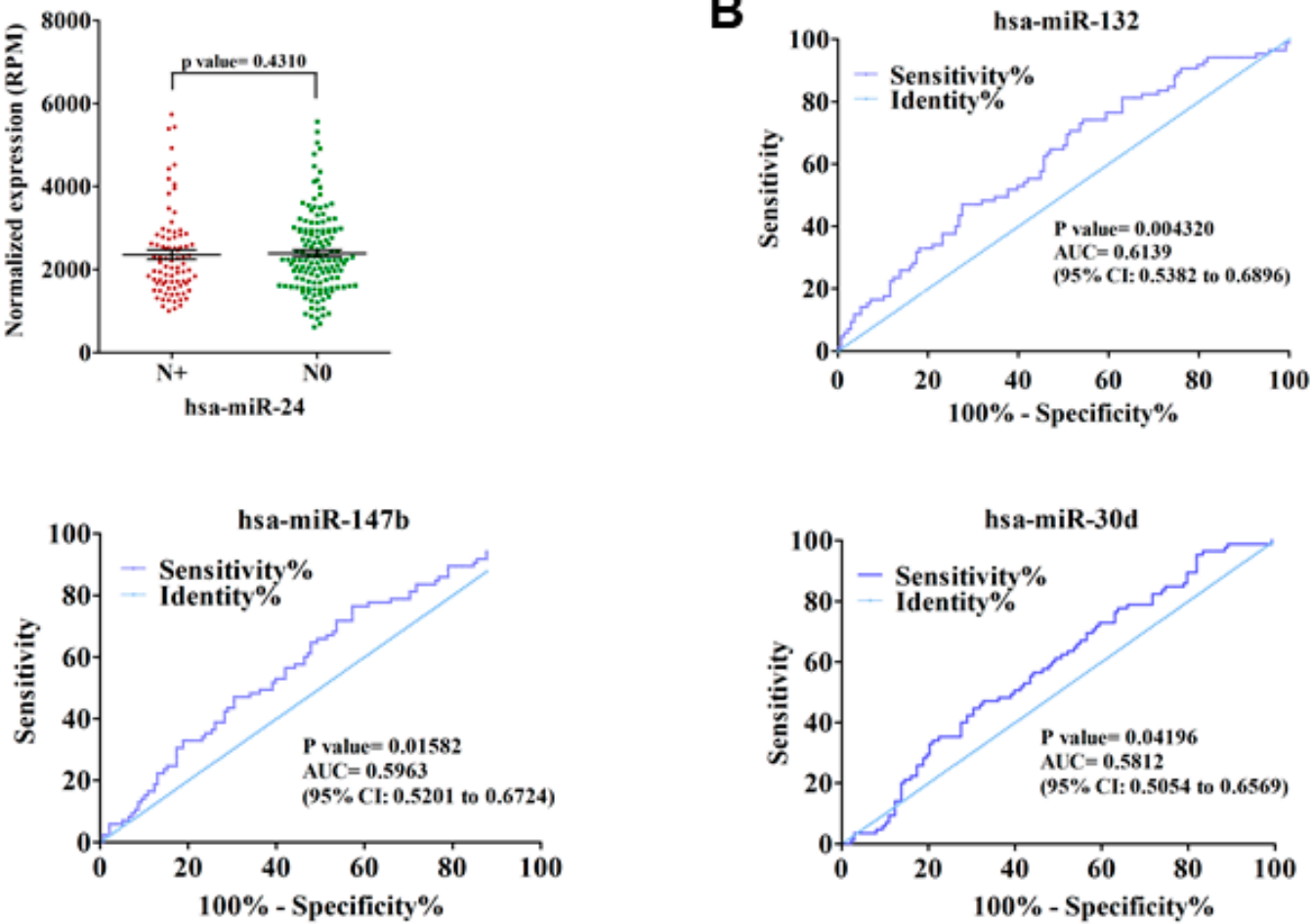

Figure 5. Mutant $\mathrm{p} 53^{\mathrm{R} 273 \mathrm{H}}$-regulated miRNAs predict lymph node metastasis (LNM) in lung adenocarcinoma patients. (A) Relative expression of seven mutant p53-regulated miRNAs in lymph node positive $\left(\mathrm{N}_{+}, n=85\right)$ and lymph node negative $\left(\mathrm{N}_{0}, n=138\right)$ patients. Scatter plots showing normalized expression (RPM) of individual miRNAs in $\mathrm{N}_{+}$and $\mathrm{N}_{0}$ group of patients. $p$-values are indicated. (B) Receiver operating characteristic (ROC) curve analyses for LNM prediction in lung adenocarcinoma patients using relative expression of $\mathrm{miR}-132$, miR-147b, and miR-30d. Area under the receiver operating characteristic curve (AUC) is shown. $p$-values are indicated. 

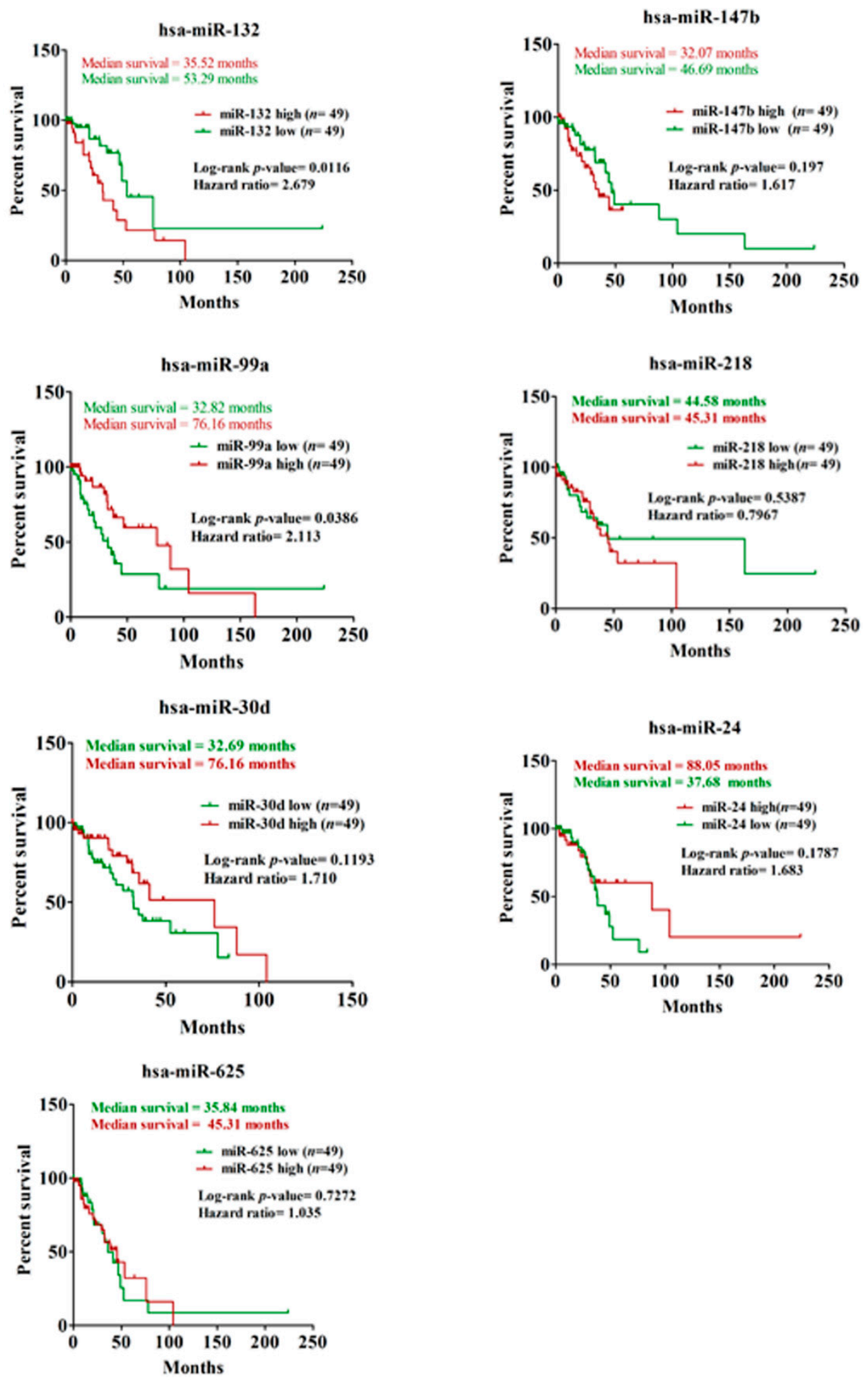

Figure 6. Mutant $\mathrm{p} 53^{\mathrm{R} 273 \mathrm{H}}$-regulated miRNAs determine poor survival in lung adenocarcinoma patients. Kaplan-Meier analyses showing relative survival probabilities of lung adenocarcinoma patients with high ( $\geq 75$ th percentile) and low ( $\leq 25$ th percentile) expression of the individual mutant p53-regulated miRNAs. Log-rank $p$-value, hazard ratio, and median survival time are indicated. $n$ indicates the number of patients in respective miRNA low and high groups. 
Among the five down-regulated miRNAs, the expression of only miR-30d was found to be significantly ( $p$-value $\leq 0.05$ ) low in $\mathrm{N}_{+}$patients when compared to those without lymph node involvement (Figure 5A). The observation suggests a negative role of miR-30d in lymphatic metastasis during lung cancer progression. We validated these three miRNAs (miR-132, miR-147b, and miR-30d) in predicting lung cancer lymph node metastasis while using ROC (Receiver operating characteristic) curve analysis to further strengthen these results. For all three miRNAs, the LNM prediction accuracy was found to be statistically significant ( $p$-value $\leq 0.05)$ with an area under the curve $($ AUC) $>$ $0.5(95 \% \mathrm{CI})$ (Figure 5B), thereby suggesting these miRNAs as significant predictors of LNM in lung adenocarcinoma. To further investigate the prognostic significance of mutant p53-regulated miRNAs in lung adenocarcinoma, we assessed their correlation with patients' overall survival using Kaplan-Meier survival analysis. Among the seven miRNAs, expression of miR-132 and miR-99a were found to be significantly (Log-rank $p$-value $\leq 0.05$ ) associated with patients' survival (Figure 6 and Supplemental Table S4). The analysis showed poor overall survival in patients with high miR-132 expression (Median survival, 35.52 months; Hazard ratio, 2.679), as well as in those with low miR-99a expression (Median survival, 32.82 months; Hazard ratio, 2.113). Taken together, our analyses suggest a significant contribution of mutant p53-regulated miRNAs in determining the clinical outcome in lung adenocarcinoma patients.

\subsection{Mutant p53-Regulated MiR-194 and MiR-378a Predict EMT Phenotype in Cancer Cells}

GOF mutant p53 promotes EMT by modulating the expression of genes that are involved in cell adhesion, invasion, and migration [42-44]. Moreover, miRNAs are implicated in mutant p53-driven EMT process [26,45]. Hence, we investigated whether mutant $\mathrm{p} 53^{\mathrm{R} 273 \mathrm{H}}$-regulated miRNAs that were obtained in our study could also predict EMT in cancer cells. To this end, we conducted an integrated analysis using the NCI-60 miRNA expression dataset [46]. Among the 47 differentially regulated miRNAs identified in the present study, information on 37 were available in the NCI-60 dataset (GSE26375). Based on the E-Cadherin/Vimentin expression ratio [36], the NCI-60 cell lines were first divided into epithelial and mesenchymal cell types and differential expression analysis for the 37 miRNAs was subsequently carried out between these two groups. Two miRNAs, miR-194 and miR-378a, were found to be significantly ( $p$-value $\leq 0.05$ ) down-regulated in the mesenchymal group as compared to that of epithelial cells (Figure 7). Previous reports also suggested the involvement of these two miRNAs in EMT and cancer metastasis [47,48]. Furthermore, negative regulation of miR-194 by mutant p53 has also been shown in endometrial cancer cells [26]. In view of these previous reports, the results of our analyses point towards an important role of mir-194 and miR-378a in mediating the GOF mutant p53-driven EMT process in cancer cells.

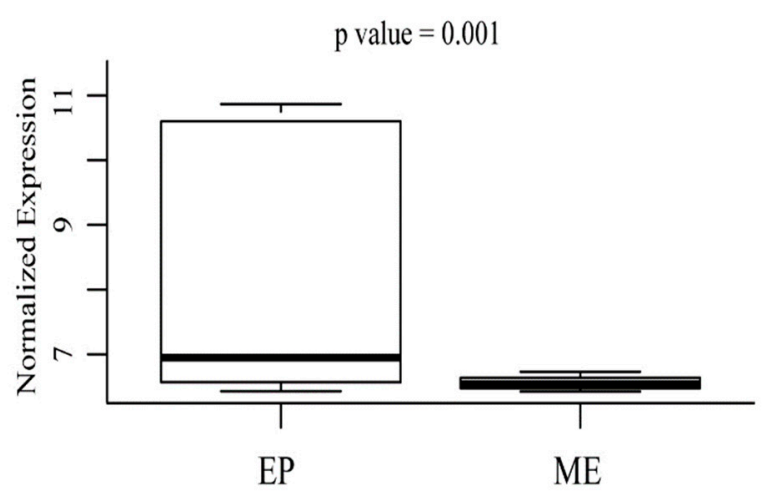

hsa-miR-194

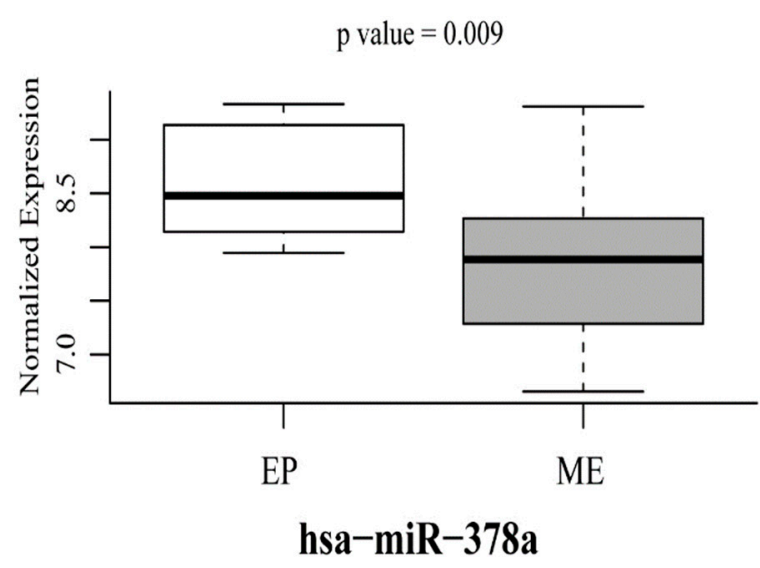

hsa-miR-378a

Figure 7. GOF Mutant $\mathrm{p} 53^{\mathrm{R} 273 \mathrm{H}}$-regulated miR-194 and miR-378a is down-regulated in NCI-60 cell lines with mesenchymal phenotype. Box-Whisker plots showing relative expression of miR-194 and miR-378a in mesenchymal and epithelial groups of NCI-60 cell lines. $p$-values are indicated. 


\subsection{Discovery of a Novel MiRNA}

Next-generation deep sequencing coupled with high throughput bioinformatic analyses enables researchers to discover novel miRNAs with high sensitivity and specificity [49]. Here, while using the sequencing reads that did not align to the miRbase (miRBase20; http:/www.mirbase.org/), we tried to identify candidate sequences that have potential precursor miRNA hairpin structures around their genomic coordinates. We identified three putative hairpin precursor miRNAs. One of them is in the intergenic region of chromosome 1 (chr1:168239822-168239840), while the other two overlaps with the tRNA-Ile-ATT (chr6:27,636,363-27,636,381) and tRNA-Asp-GAY (chr12:96,429,800-96,429,817). These two candidates might have been falsely determined and they could be part of the degraded tRNA. However tRNA-derived RNA fragments [50] and miRNAs [51] are also being reported in the literature. The sequence that was mapped to the intergenic region of the genome was considered for further validation. The secondary structure of its precursor miRNA showed a characteristic stem-loop structure (Figure $8 \mathrm{~A}$ ) with $\Delta \mathrm{G}=-27.40 \mathrm{kcal} / \mathrm{mol}$ and the mature sequence mainly lies on the stem region. Interestingly, the novel miRNA, designated as "miR-X" was found to be homologous to the efu-miR-9277 of bat, which suggests its possible existence. Furthermore, we have used the publicly available dataset (GSE33858) of lung adenocarcinoma, squamous cell carcinoma, and corresponding noncancerous tissue samples, and evaluated the expression profile of miR-X. Integrative Genomics Viewer of the primary aligned reads across the miR- $X$ region showed the expression of $\mathrm{miR}-X$ in some of lung adenocarcinoma, squamous cell carcinoma, and adjacent noncancerous tissues (Supplemental Figure S3). miR-X was found to be upregulated in some of the adenocarcinoma and squamous cell carcinoma tissues as compared to the corresponding adjacent noncancerous tissues of the same patients but was not significant at the level of $p=0.05$ (Figure $8 \mathrm{~B}$ ). To explore the biological functions of miR-X, we predicted its downstream targets while using miRanda [31] and identified several putative target genes (Supplemental Table S5). Subsequent pathway analysis of the predicted target genes showed significant enrichment of important biological pathways (Supplemental Table S6). Moreover, network analyses of the target genes indicated the possible involvement of miR-X in crucial cellular processes that are commonly deregulated in cancer, including cell growth and proliferation; cellular assembly and organization, DNA replication, recombination and repair, and cell-to-cell signaling (Supplemental Table S6).

\subsection{Novel miR-X Promotes Oncogenic Properties in Lung Adenocarcinoma Cells}

Next, we aimed to investigate the expression levels of the newly identified miRNA in cancer cells harboring GOF mutant $\mathrm{p} 53$. To this aim, we compared the relative expression of miR-X between H1299/EV and H1299/mutant p53 ${ }^{\mathrm{R} 273 \mathrm{H}}$ stable cell lines while using qRT-PCR. We found significant up-regulation of miR-X in H1299 cells harboring mutant $\mathrm{p} 53^{\mathrm{R} 273 \mathrm{H}}$ when compared to the control cells (Figure 9A). To further asses its possible biological functions, we investigated the cellular effect of miR-X overexpression in different cell-based assays. For this, we cloned the newly identified miRNA into pRNA.U6 miRNA expression vector and subsequently overexpressed in H1299 cells (Figure 9B). The ectopic expression of miR-X led to a marked increase in the proliferation rate of H1299 cells, as measured by WST-1 cell proliferation assay (Figure 9C), which was one of the most significantly enriched networks for the miR-X target genes (Supplemental Table S6). We overexpressed miR-X in another lung cancer cell line A549 to further validate this observation. A549 cells overexpressing miR-X also showed significantly enhanced proliferation as compared to the control empty vector transfected cells (Figure 9C). Consistent with these results, we observed significantly higher percentage of Ki67 positive cells in both $\mathrm{H} 1299$ and A549 cells upon miR-X overexpression, thereby confirming its role in promoting lung cancer cell proliferation (Figure 9D,E). The growth promoting effect of miR-X was further recapitulated in subsequent colony formation assay, where ectopic expression of the miRNA clearly promoted the colony forming ability in H1299 cells (Figure 9F). We performed a monolayer wound healing assay to determine whether miR-X could also promote cancer cells migration. When compared to the empty vector-transfected H1299 cells, cells overexpressing miR-X was found to migrate significantly faster, as measured by the relative distance that is covered by the cells after the 
scratch was introduced (Figure 9G). Together, these results suggest a potential role of miR-X in driving oncogenic phenotypes.

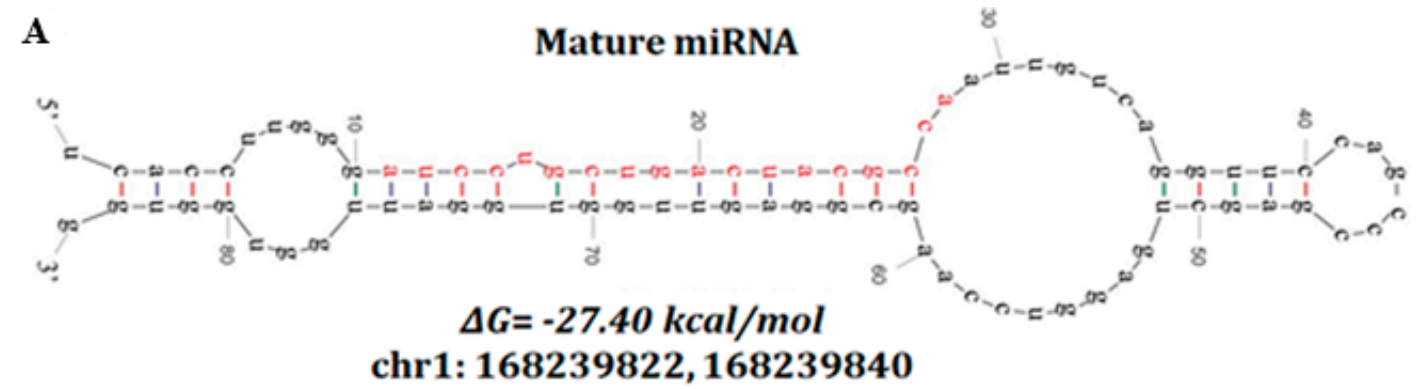

B

miR-X expression in Adenocarcinoma Lung Cancer

miR.X expression in Squamous Lung Cancer
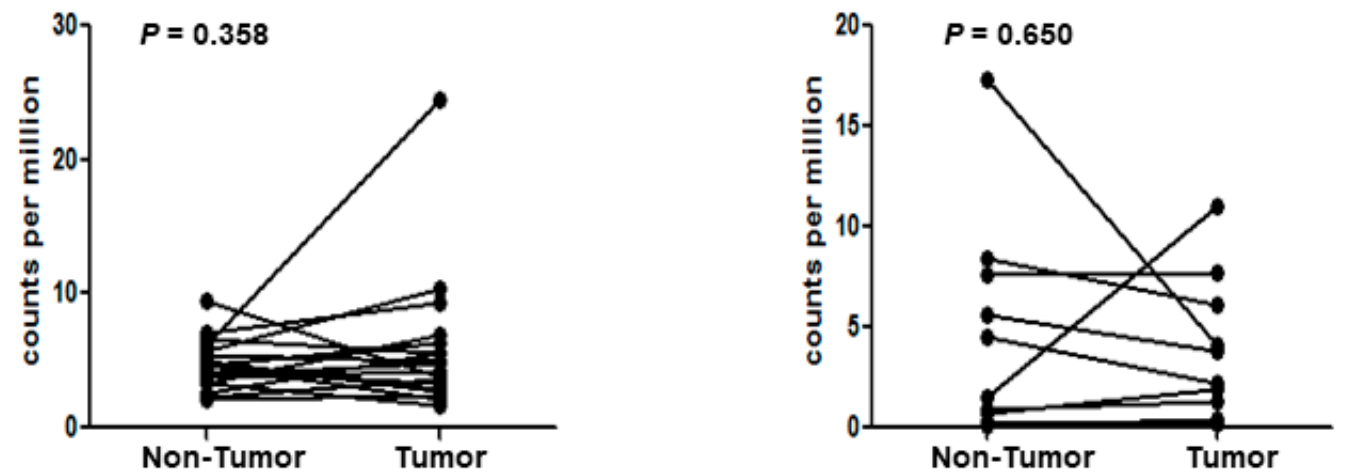

Figure 8. Discovery of the novel miRNA. (A) Stem-loop structure of the novel miRNA (miR-X) precursor predicted by mfold version 3.6. The mature miRNA sequence is shown in red. The minimum free energy (MFE; $\Delta \mathrm{G})$ of hairpin structure and the genomic coordinate of the mature sequence are indicated. (B) Symbols and line graphs showing relative expression (counts per million) levels of miR-X in paired tumor and non-tumor lung adenocarcinoma (left panel) or squamous cell carcinoma (right panel) tissues. $p$-values are indicated. 
A
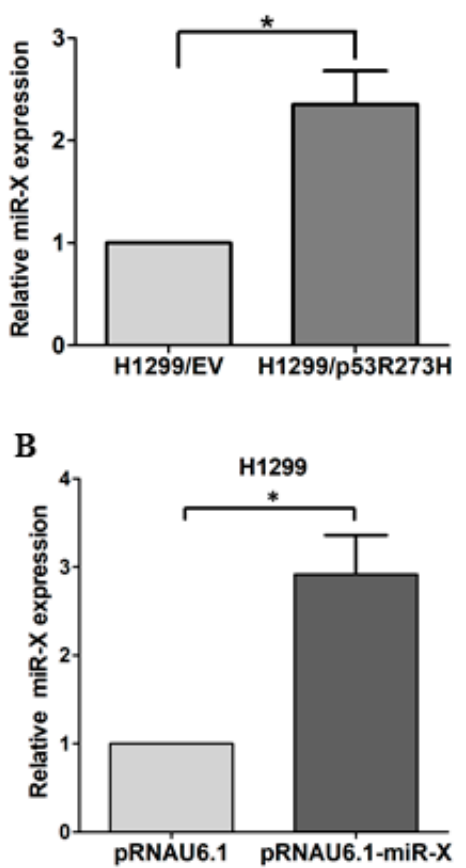

F
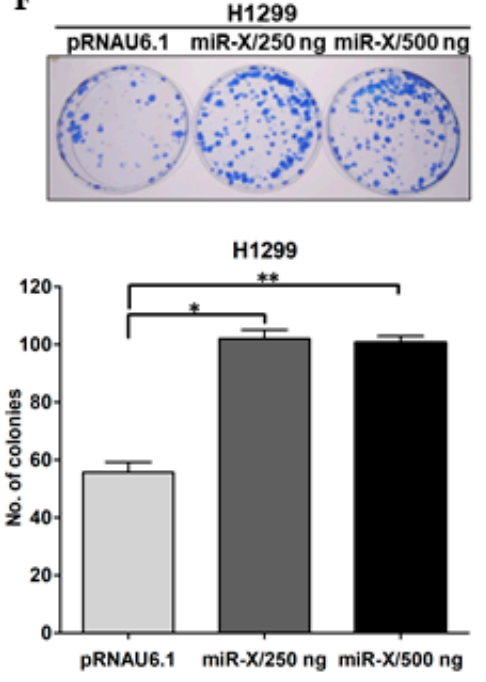

C
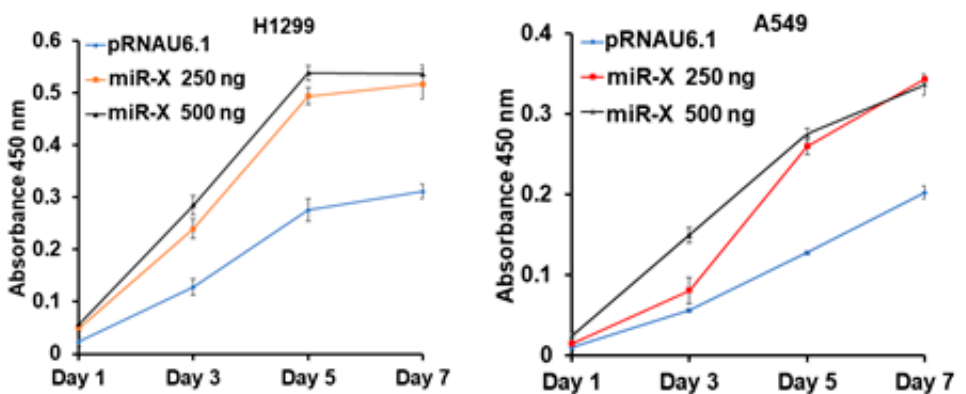

D
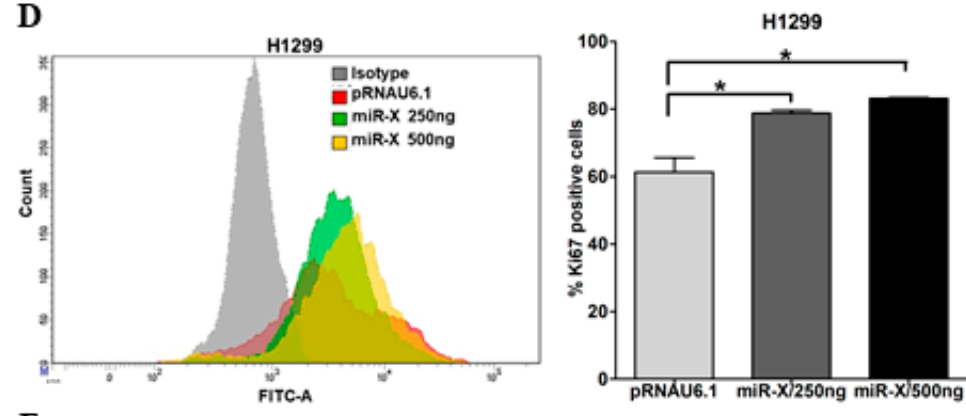

E
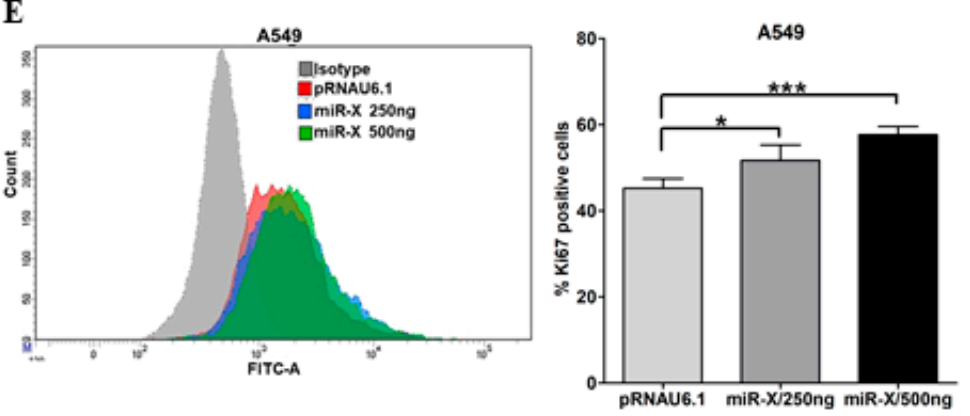

G

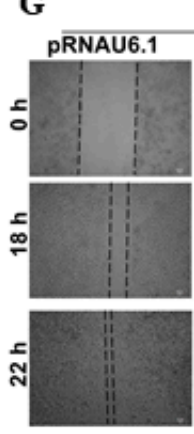

H1299
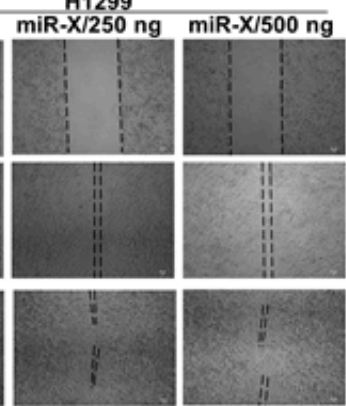

Figure 9. miR-X promotes oncogenic properties in lung cancer cells. (A) QRT-PCR data showing relative expression levels of miR-X in H1299/EV and H1299/mutant p53 ${ }^{\mathrm{R} 273 \mathrm{H}}$ stable cell lines (B) QRT-PCR data showing ectopic expression of miR-X in H1299 cells transfected with pRNAU6.1-miR-X. H1299 cells were transfected with either empty pRNAU6.1 vector or with $250 \mathrm{ng}$ of pRNAU6.1-miR-X. Forty eight hours post-transfection relative miR-X expression was evaluated using qRT-PCR. (C) Bar graphs showing relative proliferation of H1299 (left panel) and A549 (right panel) cells upon ectopic expression of miR-X as measured by WST-1 cell proliferation assay. Cells were transfected with either empty vector or miR-X expression plasmid and $16 \mathrm{~h}$ post-transfection WST-1 assay was performed for the day 1 and for the days indicated. Data represents average absorbance values at $450 \mathrm{~nm}$ of three independent experiments. (D,E) (Left panels) Histogram showing mean fluorescent intensities of empty vector and miR-X transfected H1299 (D) and A549 (E) cells stained with anti-Ki-67 antibody as measured by FACS 
analyses. (Right panels) Bar graphs showing relative percentages of Ki-67 positive H1299 (D) and A549 (E) cells transfected with control empty vector or with miR-X expression plasmid. (F) (Upper panel) Representative images of colonies formed by H1299 cells upon miR-X overexpression in clonogenic assay. (Lower panel) Quantification of the data that were obtained from the clonogenic assay. (G) (Left panel) Representative images of wound healing assay performed in $\mathrm{H} 1299$ cells transfected with either empty vector or miR-X expression plasmid. Images were captured at $0 \mathrm{~h}, 18 \mathrm{~h}$, and $22 \mathrm{~h}$ after the scratch was introduced. (Right panel) Bar graph showing relative percentage of distance covered by the cells through $18 \mathrm{~h}$ post-scratch. Data represent mean \pm s.d.; $n=3$; two-tailed Student's $t$-test: ${ }^{*} p<0.05$, ** $p<0.01,{ }^{* * *} p<0.001$.

\section{Discussion}

GOF mutant p53, an oncogenic transcription factor, transactivates or represses a diverse set of genes to promote tumorigenesis $[3,5,9,35,52,53]$. Beside this, the regulation of cellular miRNAs is also implicated in different oncogenic phenotypes conferred by mutant p53 [24-29]. Therefore, identification of miRNAs regulated by mutant $\mathrm{p} 53$ on a global scale would provide further insights into the cellular pathways and biological functions that are governed by GOF mutant p53 in the context of tumorigenesis. In this study, we explored GOF mutant $\mathrm{p} 53^{\mathrm{R} 273 \mathrm{H}}$-regulated miRnome in lung adenocarcinoma cells while using small RNA deep sequencing. Subsequent target gene prediction and network enrichment analyses revealed that mutant $\mathrm{p} 53^{\mathrm{R} 273 \mathrm{H}}$-regulated miRNAs interact with several crucial genes and molecular pathways that are implicated in tumorigenesis. Enrichment of p53 in the miRNA regulatory network indicates that specific miRNAs (miR-99a, -132, -140, -17, -194, and -296) are most likely to be regulated by both wild-type and mutant forms of p53, as also suggested by previous studies [23,54-56]. Our analyses also predicted molecular interactions between mutant p53-regulated miRNAs and cancer metastasis-associated genes TGFBI, EPHB6, and insulin signaling, a pathway that is commonly deregulated in cancer [57,58]. Integrated analysis of miRNA and mRNA expression profiles further identified potential miRNA-mRNA modules that might be critical in mediating mutant p53 gain-of-functions in cancer cells.

We validated our cell-based data in the TCGA lung adenocarcinoma patient dataset to further investigate the prognostic value of the mutant $\mathrm{p} 53^{\mathrm{R} 273 \mathrm{H}}$-regulated miRNAs. Approximately $30 \%$ of patients in the TCGA dataset had missense mutations in TP53, whereas $16 \%$ carried the truncated version of the protein. High TP53 mutation frequencies in lung cancer patients clearly indicate the importance of studying the mutant p53-specific regulation of miRNAs. Eighteen miRNAs that were detected in our cell line study showed similar expression patterns in the patient samples, however, only seven of them were statistically significant. It is quite reasonable to consider that different mutant p53 proteins might differentially regulate miRNAs that were obtained from our cell line study since the dataset contains patients with different types of TP53 mutation. Genetic heterogeneity among patients might also account for the limited agreement of our cell line data with the TCGA dataset. Several studies evidenced the association of tumor metastasis with TP53 mutations in different types of cancers [40,41]. Here, we investigated the possible contribution of mutant p53 ${ }^{\mathrm{R} 273 \mathrm{H}}$-regulated miRNAs in predicting lymph node metastasis in lung adenocarcinoma patients by systematic bioinformatics analyses of the TCGA LUAD dataset. Among the seven mutant $\mathrm{p} 53^{\mathrm{R} 273 \mathrm{H}}$-regulated miRNAs, the expression of miR-132, $-147 \mathrm{~b}$, and $-30 \mathrm{~d}$ was found to be significantly correlated with the LNM status of the patients. ROC curve analysis further confirmed that these miRNAs are significant predictors of LNM in NSCLC. We hypothesize that mutant p53 modulates the expression of these miRNAs to drive lymph node metastasis in NSCLC, as TP53 mutations generally precede node metastasis in lung cancer [15]. However, further studies are required to ascertain the contribution of these miRNAs in node metastasis. This has been suggested that miRNAs are potential contributors to mutant p53-driven EMT phenotype [26]. In agreement with this, our integrated analyses of the NCI-60 miRNA expression dataset identified mutant $\mathrm{p} 53^{\mathrm{R} 273 \mathrm{H}}$-regulated miR-194 and miR-378a as potential determinants of EMT 
in cancer cell lines. Taken together, our analyses clearly indicate an important role of cellular miRNAs in mediating diverse oncogenic properties of GOF mutant p53 in human cancer.

It is anticipated that many more miRNAs are yet to be discovered than presently annotated in public repositories [59]. In this study, we discovered a novel miRNA from the unmapped sequence reads of the genome-wide small RNA sequencing data. Further analyses using publicly available small RNA sequencing dataset of lung cancer tissues showed enough primary reads to be precisely aligned to the genomic region of the newly identified miRNA. This suggests that miR-X, although not identified earlier, was present in lung cancer and adjacent benign lung tissues. The precursor miR-X overlaps with two LTRs of ERVL family (HERVL18-int and LTR18A), while 13 bases of the mature miR-X overlap with one of the LTRs and remaining five bases are in the non-LTR region. However, it should be noted that $\sim 20 \%$ of the human miRNAs are reported to have complete or partial overlap with transposable elements, and many of them are proposed to be derived from transposable elements, including LTR, LINE, and SINE [60-62]. Target prediction and pathway analyses revealed that the novel miRNA targets a wide array of genes that are involved in different signaling pathways. Subsequent cell-based assays further indicated that the newly identified miRNA could promote oncogenic phenotypes, including cancer cell proliferation, clonogenicity, and migration, which suggests its potential role in oncogenesis. Increased expression of the novel miRNA in GOF mutant p53 expressing cancer cells further suggests a plausible role of this miRNA in mediating mutant p53-driven tumorigenesis. However, we could not see any significant difference in miR-X expression between adjacent normal and lung tumor tissues, at least in the publicly available small RNA sequencing dataset that we analyzed in the present study. This might be attributed to the tumor heterogeneity or insufficient number of tumor samples analyzed. Although further functional studies are required to fully characterize the novel miRNA, it holds the potential to be included in the growing list of cellular miRNAs. Collectively, our study provides an important framework for further research on understanding the molecular mechanism of GOF mutant p53-driven tumorigenesis and identified a specific miRNA signature that might be useful in predicting NSCLC prognosis.

Supplementary Materials: The following are available online at http://www.mdpi.com/2073-4425/10/11/852/ s1. Figure S1: Quality control of small RNA sequencing data. Figure S2: Regulatory networks of mutant p53 ${ }^{\mathrm{R} 273 \mathrm{H}}$-regulated miRNAs. Figure S3: Small RNA seq mapped reads alignment around miR-X region in Integrative Genomics Viewer (IGV). Table S1: IPA analysis of differentially expressed miRNAs between H1299/EV and H1299/mutantp53R273H cells. Table S2: miRNAs and their target mRNAs with opposite expression pattern in H1299/mutant p53R273H cells. Table S3: Normalized read counts of seven mutant p53R273H-regulated miRNAs in lymph node positive and lymph node negative TCGA LUAD patients. Table S4: Survival data and normalized read counts of seven mutant p53 R273H-regulated miRNAs in TCGA LUAD patients. Table S5: Targets of Novel miR-X predicted by miRanda. Table S6: Pathway analysis of Novel miR-X target genes in IPA. Table S7: Primers used for qRT-PCR validation.

Author Contributions: Conceptualization, A.D. and S.R.; methodology, A.D., P.D., S.D., S.G. and D.G.; software, P.D., S.D., R.C., and S.R.; validation, A.D., P.D. and R.C.; formal analysis, A.D., P.D., S.D., S.K.A. and R.C.; investigation, A.D., R.C. and S.R.; resources, S.R. and R.C.; data curation, A.D., S.D., P.D. and R.C., writing-Original draft preparation, A.D., P.D., and S.R.; writing-Review and editing, A.D., R.C. and S.R., supervision, S.R.; project administration, S.R..; funding acquisition, R.C. and S.R.

Funding: This work was supported by CSIR-Mayo Clinic Collaboration for Innovation and Translational Research Grant CMPP-08, CSIR-NMITLI project TLP 0007 and J C Bose Fellowship (JCB/2017/000005) awarded to S. Roychoudhury. R. Chatterjee is supported by the intramural funding from Indian Statistical Institute and extramural funding from CSIR Grant 27(0306)/14//EMR-II.

Acknowledgments: We thank Varda Rotter (Weizmann Institute of Science, Rehovot, Israel) for kindly providing us with the H1299/EV cell line. We thank David Callen (Centre for Personalized Cancer Medicine, School of Medicine, The University of Adelaide, Australia) for kindly providing us with the mutant $\mathrm{p} 53^{\mathrm{R} 273 \mathrm{H}}$ transcriptome dataset in the H1299 cell line. We thank Atreyee Saha (Life Technologies, India) for helping us with Ion PGM ${ }^{\mathrm{TM}}$ sequencing facility. We thank Neha Garg (Life Technologies, India) Joyeeta Chakraborty and Sayantan Laha (Indian Statistical Institute, Kolkata, India) for their valuable suggestions in data analysis.

Conflicts of Interest: The authors declare no competing interests. 


\section{References}

1. Hollstein, M.; Sidransky, D.; Vogelstein, B.; Harris, C.C. p53 mutations in human cancers. Science 1991, 253, 49-53. [CrossRef]

2. Petitjean, A.; Achatz, M.I.; Borresen-Dale, A.L.; Hainaut, P.; Olivier, M. TP53 mutations in human cancers: Functional selection and impact on cancer prognosis and outcomes. Oncogene 2007, 26, 2157-2165. [CrossRef]

3. Muller, P.A.; Vousden, K.H. p53 mutations in cancer. Nat. Cell Biol. 2013, 15, 2-8. [CrossRef]

4. Olivier, M.; Hollstein, M.; Hainaut, P. TP53 mutations in human cancers: Origins, consequences, and clinical use. Cold Spring Harb. Perspect. Biol. 2010, 2, a001008. [CrossRef]

5. Oren, M.; Rotter, V. Mutant p53 gain-of-function in cancer. Cold Spring Harb. Perspect. Biol. 2010, 2, a001107. [CrossRef]

6. Datta, A.; Ghatak, D.; Das, S.; Banerjee, T.; Paul, A.; Butti, R.; Gorain, M.; Ghuwalewala, S.; Roychowdhury, A.; Alam, S.K.; et al. p53 gain-of-function mutations increase Cdc7-dependent replication initiation. EMBO Rep. 2017, 18, 2030-2050. [CrossRef]

7. Singh, S.; Vaughan, C.A.; Frum, R.A.; Grossman, S.R.; Deb, S.; Palit Deb, S. Mutant p53 establishes targetable tumor dependency by promoting unscheduled replication. J. Clin. Investig. 2017, 127, 1839-1855. [CrossRef]

8. Bajaj, S.; Alam, S.K.; Roy, K.S.; Datta, A.; Nath, S.; Roychoudhury, S. E2 Ubiquitin-conjugating Enzyme, UBE2C Gene, Is Reciprocally Regulated by Wild-type and Gain-of-Function Mutant p53. J. Biol. Chem. 2016, 291, 14231-14247. [CrossRef]

9. Strano, S.; Dell'Orso, S.; Di Agostino, S.; Fontemaggi, G.; Sacchi, A.; Blandino, G. Mutant p53: An oncogenic transcription factor. Oncogene 2007, 26, 2212-2219. [CrossRef]

10. Gaiddon, C.; Lokshin, M.; Ahn, J.; Zhang, T.; Prives, C. A subset of tumor-derived mutant forms of p53 down-regulate p63 and p73 through a direct interaction with the p53 core domain. Mol. Cell. Biol. 2001, 21, 1874-1887. [CrossRef]

11. Strano, S.; Fontemaggi, G.; Costanzo, A.; Rizzo, M.G.; Monti, O.; Baccarini, A.; Del Sal, G.; Levrero, M.; Sacchi, A.; Oren, M.; et al. Physical interaction with human tumor-derived p53 mutants inhibits p63 activities. J. Biol. Chem. 2002, 277, 18817-18826. [CrossRef]

12. Freed-Pastor, W.A.; Prives, C. Mutant p53: One name, many proteins. Genes Dev. 2012, 26, 1268-1286. [CrossRef]

13. Parkin, D.M.; Bray, F.I.; Devesa, S.S. Cancer burden in the year 2000. The global picture. Eur. J. Cancer 2001, 37, S4-S66. [CrossRef]

14. Ahrendt, S.A.; Hu, Y.; Buta, M.; McDermott, M.P.; Benoit, N.; Yang, S.C.; Wu, L.; Sidransky, D. p53 mutations and survival in stage I non-small-cell lung cancer: Results of a prospective study. J. Natl. Cancer Inst. 2003, 95, 961-970. [CrossRef]

15. Mogi, A.; Kuwano, H. TP53 mutations in nonsmall cell lung cancer. J. Biomed. Biotechnol. 2011, $2011,583929$. [CrossRef]

16. Mitsudomi, T.; Hamajima, N.; Ogawa, M.; Takahashi, T. Prognostic significance of p53 alterations in patients with non-small cell lung cancer: A meta-analysis. Clin. Cancer Res. 2000, 6, 4055-4063.

17. Scian, M.J.; Stagliano, K.E.; Anderson, M.A.; Hassan, S.; Bowman, M.; Miles, M.F.; Deb, S.P.; Deb, S. Tumor-derived p53 mutants induce NF-kappaB2 gene expression. Mol. Cell. Biol. 2005, 25, 10097-10110. [CrossRef]

18. Yeudall, W.A.; Vaughan, C.A.; Miyazaki, H.; Ramamoorthy, M.; Choi, M.Y.; Chapman, C.G.; Wang, H.; Black, E.; Bulysheva, A.A.; Deb, S.P.; et al. Gain-of-function mutant p53 upregulates CXC chemokines and enhances cell migration. Carcinogenesis 2012, 33, 442-451. [CrossRef]

19. Muller, P.A.; Vousden, K.H. Mutant p53 in cancer: new functions and therapeutic opportunities. Cancer Cell 2014, 25, 304-317. [CrossRef]

20. Garzon, R.; Calin, G.A.; Croce, C.M. MicroRNAs in Cancer. Annu. Rev. Med. 2009, 60, 167-179. [CrossRef]

21. Feng, Z.; Zhang, C.; Wu, R.; Hu, W. Tumor suppressor p53 meets microRNAs. J. Mol. Cell Biol. 2011, 3, 44-50. [CrossRef]

22. Chang, T.C.; Wentzel, E.A.; Kent, O.A.; Ramachandran, K.; Mullendore, M.; Lee, K.H.; Feldmann, G.; Yamakuchi, M.; Ferlito, M.; Lowenstein, C.J.; et al. Transactivation of miR-34a by p53 broadly influences gene expression and promotes apoptosis. Mol. Cell 2007, 26, 745-752. [CrossRef] 
23. Tarasov, V.; Jung, P.; Verdoodt, B.; Lodygin, D.; Epanchintsev, A.; Menssen, A.; Meister, G.; Hermeking, H. Differential regulation of microRNAs by p53 revealed by massively parallel sequencing: Mir-34a is a p53 target that induces apoptosis and G1-arrest. Cell Cycle 2007, 6, 1586-1593. [CrossRef]

24. Donzelli, S.; Fontemaggi, G.; Fazi, F.; Di Agostino, S.; Padula, F.; Biagioni, F.; Muti, P.; Strano, S.; Blandino, G. MicroRNA-128-2 targets the transcriptional repressor E2F5 enhancing mutant p53 gain of function. Cell Death Differ. 2012, 19, 1038-1048. [CrossRef]

25. Masciarelli, S.; Fontemaggi, G.; Di Agostino, S.; Donzelli, S.; Carcarino, E.; Strano, S.; Blandino, G. Gain-of-function mutant p53 downregulates miR-223 contributing to chemoresistance of cultured tumor cells. Oncogene 2014, 33, 1601-1608. [CrossRef]

26. Dong, P.; Karaayvaz, M.; Jia, N.; Kaneuchi, M.; Hamada, J.; Watari, H.; Sudo, S.; Ju, J.; Sakuragi, N. Mutant p53 gain-of-function induces epithelial-mesenchymal transition through modulation of the miR-130b-ZEB1 axis. Oncogene 2013, 32, 3286-3295. [CrossRef]

27. Neilsen, P.M.; Noll, J.E.; Mattiske, S.; Bracken, C.P.; Gregory, P.A.; Schulz, R.B.; Lim, S.P.; Kumar, R.; Suetani, R.J.; Goodall, G.J.; et al. Mutant p53 drives invasion in breast tumors through up-regulation of miR-155. Oncogene 2013, 32, 2992-3000. [CrossRef]

28. Subramanian, M.; Francis, P.; Bilke, S.; Li, X.L.; Hara, T.; Lu, X.; Jones, M.F.; Walker, R.L.; Zhu, Y.; Pineda, M.; et al. A mutant p53/let-7i-axis-regulated gene network drives cell migration, invasion and metastasis. Oncogene 2015, 34, 1094-1104. [CrossRef]

29. Wang, W.; Cheng, B.; Miao, L.; Mei, Y.; Wu, M. Mutant p53-R273H gains new function in sustained activation of EGFR signaling via suppressing miR-27a expression. Cell Death Dis. 2013, 4, e574. [CrossRef]

30. Chen, C.; Ridzon, D.A.; Broomer, A.J.; Zhou, Z.; Lee, D.H.; Nguyen, J.T.; Barbisin, M.; Xu, N.L.; Mahuvakar, V.R.; Andersen, M.R.; et al. Real-time quantification of microRNAs by stem-loop RT-PCR. Nucleic Acids Res. 2005, 33, e179. [CrossRef]

31. Enright, A.J.; John, B.; Gaul, U.; Tuschl, T.; Sander, C.; Marks, D.S. MicroRNA targets in Drosophila. Genome Biol. 2003, 5, R1. [CrossRef] [PubMed]

32. Cancer Genome Atlas Research Network. Comprehensive molecular profiling of lung adenocarcinoma. Nature 2014, 511, 543-550. [CrossRef] [PubMed]

33. Cerami, E.; Gao, J.; Dogrusoz, U.; Gross, B.E.; Sumer, S.O.; Aksoy, B.A.; Jacobsen, A.; Byrne, C.J.; Heuer, M.L.; Larsson, E.; et al. The cBio cancer genomics portal: An open platform for exploring multidimensional cancer genomics data. Cancer Discov. 2012, 2, 401-404. [CrossRef]

34. Gao, J.; Aksoy, B.A.; Dogrusoz, U.; Dresdner, G.; Gross, B.; Sumer, S.O.; Sun, Y.; Jacobsen, A.; Sinha, R.; Larsson, E.; et al. Integrative analysis of complex cancer genomics and clinical profiles using the cBioPortal. Sci. Signal. 2013, 6. [CrossRef]

35. Van Oijen, M.G.; Slootweg, P.J. Gain-of-function mutations in the tumor suppressor gene p53. Clin. Cancer Res. 2000, 6, 2138-2145.

36. Park, S.M.; Gaur, A.B.; Lengyel, E.; Peter, M.E. The miR-200 family determines the epithelial phenotype of cancer cells by targeting the E-cadherin repressors ZEB1 and ZEB2. Genes Dev. 2008, 22, 894-907. [CrossRef]

37. Anders, S.; Pyl, P.T.; Huber, W. HTSeq-A Python framework to work with high-throughput sequencing data. Bioinformatics 2015, 31, 166-169. [CrossRef]

38. Anders, S.; Huber, W. Differential expression analysis for sequence count data. Genome Biol. 2010, 11, R106. [CrossRef]

39. Neilsen, P.M.; Noll, J.E.; Suetani, R.J.; Schulz, R.B.; Al-Ejeh, F.; Evdokiou, A.; Lane, D.P.; Callen, D.F. Mutant p53 uses p63 as a molecular chaperone to alter gene expression and induce a pro-invasive secretome. Oncotarget 2011, 2, 1203-1217. [CrossRef]

40. Campling, B.G.; el-Deiry, W.S. Clinical implications of p53 mutations in lung cancer. Methods Mol. Med. 2003, 75, 53-77. [CrossRef]

41. Powell, E.; Piwnica-Worms, D.; Piwnica-Worms, H. Contribution of p53 to metastasis. Cancer Discov. 2014, 4, 405-414. [CrossRef]

42. Kogan-Sakin, I.; Tabach, Y.; Buganim, Y.; Molchadsky, A.; Solomon, H.; Madar, S.; Kamer, I.; Stambolsky, P.; Shelly, A.; Goldfinger, N.; et al. Mutant p53(R175H) upregulates Twist1 expression and promotes epithelial-mesenchymal transition in immortalized prostate cells. Cell Death Differ. 2011, 18, 271-281. [CrossRef] 
43. Muller, P.A.; Vousden, K.H.; Norman, J.C. p53 and its mutants in tumor cell migration and invasion. J. Cell Biol. 2011, 192, 209-218. [CrossRef]

44. Roger, L.; Jullien, L.; Gire, V.; Roux, P. Gain of oncogenic function of p53 mutants regulates E-cadherin expression uncoupled from cell invasion in colon cancer cells. J. Cell Sci. 2010, 123, 1295-1305. [CrossRef]

45. Chang, C.J.; Chao, C.H.; Xia, W.; Yang, J.Y.; Xiong, Y.; Li, C.W.; Yu, W.H.; Rehman, S.K.; Hsu, J.L.; Lee, H.H.; et al. p53 regulates epithelial-mesenchymal transition and stem cell properties through modulating miRNAs. Nat. Cell Biol. 2011, 13, 317-323. [CrossRef]

46. Sokilde, R.; Kaczkowski, B.; Podolska, A.; Cirera, S.; Gorodkin, J.; Moller, S.; Litman, T. Global microRNA analysis of the NCI-60 cancer cell panel. Mol. Cancer Ther. 2011, 10, 375-384. [CrossRef]

47. Dong, P.; Kaneuchi, M.; Watari, H.; Hamada, J.; Sudo, S.; Ju, J.; Sakuragi, N. MicroRNA-194 inhibits epithelial to mesenchymal transition of endometrial cancer cells by targeting oncogene BMI-1. Mol. Cancer 2011, 10, 99. [CrossRef]

48. Zhang, G.J.; Zhou, H.; Xiao, H.X.; Li, Y.; Zhou, T. MiR-378 is an independent prognostic factor and inhibits cell growth and invasion in colorectal cancer. BMC Cancer 2014, 14, 109. [CrossRef]

49. Morin, R.D.; O'Connor, M.D.; Griffith, M.; Kuchenbauer, F.; Delaney, A.; Prabhu, A.L.; Zhao, Y.; McDonald, H.; Zeng, T.; Hirst, M.; et al. Application of massively parallel sequencing to microRNA profiling and discovery in human embryonic stem cells. Genome Res. 2008, 18, 610-621. [CrossRef]

50. Lee, Y.S.; Shibata, Y.; Malhotra, A.; Dutta, A. A novel class of small RNAs: tRNA-derived RNA fragments (tRFs). Genes Dev. 2009, 23, 2639-2649. [CrossRef]

51. Maute, R.L.; Schneider, C.; Sumazin, P.; Holmes, A.; Califano, A.; Basso, K.; Dalla-Favera, R. tRNA-derived microRNA modulates proliferation and the DNA damage response and is down-regulated in B cell lymphoma. Proc. Natl. Acad. Sci. USA 2013, 110, 1404-1409. [CrossRef] [PubMed]

52. Santoro, R.; Strano, S.; Blandino, G. Transcriptional regulation by mutant p53 and oncogenesis. Sub-Cell. Biochem. 2014, 85, 91-103. [CrossRef]

53. Datta, A.; Dey, S.; Das, P.; Alam, S.K.; Roychoudhury, S. Transcriptome profiling identifies genes and pathways deregulated upon floxuridine treatment in colorectal cancer cells harboring GOF mutant p53. Genom. Data 2016, 8, 47-51. [CrossRef]

54. Pichiorri, F.; Suh, S.S.; Rocci, A.; De Luca, L.; Taccioli, C.; Santhanam, R.; Zhou, W.; Benson, D.M., Jr.; Hofmainster, C.; Alder, H.; et al. Downregulation of p53-inducible microRNAs 192, 194, and 215 impairs the p53/MDM2 autoregulatory loop in multiple myeloma development. Cancer Cell 2010, 18, 367-381. [CrossRef]

55. Raver-Shapira, N.; Marciano, E.; Meiri, E.; Spector, Y.; Rosenfeld, N.; Moskovits, N.; Bentwich, Z.; Oren, M. Transcriptional activation of miR-34a contributes to p53-mediated apoptosis. Mol. Cell 2007, 26, 731-743. [CrossRef]

56. Shin, S.; Cha, H.J.; Lee, E.M.; Jung, J.H.; Lee, S.J.; Park, I.C.; Jin, Y.W.; An, S. MicroRNAs are significantly influenced by $\mathrm{p} 53$ and radiation in HCT116 human colon carcinoma cells. Int. J. Oncol. 2009, 34, 1645-1652.

57. Padua, D.; Massague, J. Roles of TGFbeta in metastasis. Cell Res. 2009, 19, 89-102. [CrossRef]

58. Yu, J.; Bulk, E.; Ji, P.; Hascher, A.; Tang, M.; Metzger, R.; Marra, A.; Serve, H.; Berdel, W.E.; Wiewroth, R.; et al. The EPHB6 receptor tyrosine kinase is a metastasis suppressor that is frequently silenced by promoter DNA hypermethylation in non-small cell lung cancer. Clin. Cancer Res. 2010, 16, 2275-2283. [CrossRef]

59. Boyd, D.B. Insulin and cancer. Integr. Cancer Ther. 2003, 2, 315-329. [CrossRef]

60. Londin, E.; Loher, P.; Telonis, A.G.; Quann, K.; Clark, P.; Jing, Y.; Hatzimichael, E.; Kirino, Y.; Honda, S.; Lally, M.; et al. Analysis of 13 cell types reveals evidence for the expression of numerous novel primate- and tissue-specific microRNAs. Proc. Natl. Acad. Sci. USA 2015, 112, E1106-E1115. [CrossRef]

61. Liang, C.; Li, Y.; Luo, J. A Novel Method to Detect Functional microRNA Regulatory Modules by Bicliques Merging. IEEE/ACM Trans. Comput. Biol. Bioinform. 2016, 13, 549-556. [CrossRef]

62. Qin, S.; Jin, P.; Zhou, X.; Chen, L.; Ma, F. The Role of Transposable Elements in the Origin and Evolution of MicroRNAs in Human. PLoS ONE 2015, 10, e0131365. [CrossRef]

(C) 2019 by the authors. Licensee MDPI, Basel, Switzerland. This article is an open access article distributed under the terms and conditions of the Creative Commons Attribution (CC BY) license (http://creativecommons.org/licenses/by/4.0/). 